\title{
Schiffer problem and isoparametric hypersurfaces
}

\author{
Vladimir E. Shklover
}

\begin{abstract}
The Schiffer Problem as originally stated for Euclidean spaces (and later for some symmetric spaces) is the following: Given a bounded connected open set $\Omega$ with a regular boundary and such that the complement of its closure is connected, does the existence of a solution to the Overdetermined Neumann Problem (N) imply that $\Omega$ is a ball? The same question for the Overdetermined Dirichlet Problem (D). We consider the generalization of the Schiffer Problem to an arbitrary Riemannian manifold and also the possibility of replacing the condition on the domain to be a ball by more general condition: to have a homogeneous boundary (i.e., boundary, admitting a transitive group of isometries). We prove that if $\Omega$ has a homogeneous boundary, then (N) and (D) always admit solutions (in fact, for infinitely many eigenvalues), but the converse statement is not always true. We show that in a number of spaces (symmetric and non-symmetric), many domains such that their boundaries are isoparametric hypersurfaces have eigenfunctions for (N) and (D) but fail the Schiffer Conjecture or even its generalization.

These ideas can be extended to other (essentially more complicated) overdetermined boundary value problems, including higher order equations and non-linear equations, which, in a number of important cases, may also have solutions in domains with isoparametric (and not necessarily homogeneous) boundaries. Also, a number of initial/boundary value problems for time-dependent equations with some extra boundary conditions have solutions for domains with the
\end{abstract}


above boundaries. If a time-dependent equation is non-linear and has blow-up, this blow-up occurs at the same time at all the points on the boundary.

\section{Introduction.}

Suppose $X$ is a complete, analytic Riemannian manifold and $\Omega \subset$ $X$ is an open, relatively compact, connected domain with $\partial \Omega$ connected with some regularity on $\partial \Omega\left(e . g ., C^{2+\varepsilon}\right)$. Various researchers have considered the existence of a function $u$ which satisfies one of the following overdetermined boundary value problems.

$$
\begin{cases}\Delta u+\alpha u=0, & \text { in } \Omega, \\ u=\text { constant }, \frac{\partial u}{\partial n}=0, & \text { on } \partial \Omega .\end{cases}
$$

This is the Overdetermined Neumann Problem.

$$
\begin{cases}\Delta u+\alpha u=0, & \text { in } \Omega, \\ u=0, \frac{\partial u}{\partial n}=\text { constant }, & \text { on } \partial \Omega .\end{cases}
$$

This is the Overdetermined Dirichlet Problem. The constants in (N) and (D) may be arbitrary non-zero real numbers (of course, one can assume that they equal 1).

REMARK. It is well known that if the boundary $\partial \Omega$ is not real-analytic, then neither (N) nor (D) has a solution (see [KiN], [Wi]). Therefore, without loss of generality we assume that $\partial \Omega$ is analytic and the solution is too so that the latter can be analytically extended to some neighborhood of the closure $\bar{\Omega}$.

These questions arose in connection with a number of applied problems, including variational problems (for example, minimizing an energy functional) and problems of integral geometry. A possible physical interpretation of $(\mathrm{N})$ (or (D)) is a stabilized heat distribution in the body $\Omega$ (when the heat sources are proportional to the temperature) with constant boundary temperature and no heat flow through the boundary for $(\mathrm{N})$ (or with zero boundary temperature and constant heat flow through the boundary for (D)). Also, to illustrate the importance of that, we show how an overdetermined boundary value problem may be related to Pompeiu Transform. 
Definition 1. Let $X$ be a homogeneous space as above and $\Omega$ a relatively compact domain in $X$. Let $G$ be the group of isometries of $X$. Define the Pompeiu Transform, $P_{\Omega}: C(X) \longrightarrow C(G)$ in the following way: given $f \in C(X), g \in G$, we have

$$
\left(P_{\Omega} f\right)(g)=\int_{g \Omega} f(x) d x .
$$

$\Omega$ is said to have Pompeiu Property $(\mathrm{PP})$ if $P_{\Omega}$ is injective.

Now suppose that $\Omega \subset X$ has the properties described in formulation of (N), (D). In 1983, C. Berenstein and M. Shahshahani (see [BeS]) proved the following:

Theorem. If $X$ is $\mathbb{R}^{n}$ or non-compact irreducible symmetric space of rank 1 , then $\Omega$ fails $(\mathrm{PP})$ if and only if $(\mathrm{N})$ has a solution with $\alpha>0$.

If $X$ is an arbitrary homogeneous space, we have a weaker statement, namely:

Theorem. If $(\mathrm{N})$ has a solution with $\alpha>0$ belonging to the global spectrum of $X$ (for the Laplace-Beltrami operator), then $\Omega$ fails (PP).

The Schiffer Conjecture (originally formulated for $X=\mathbb{R}^{n}$ ) is that existence of a solution to $(\mathrm{N})$ implies that $\Omega$ is a metric ball. So far, there are no counterexamples to the Schiffer Conjecture (SC) in $\mathbb{R}^{n}$ or non-compact irreducible symmetric spaces of the first rank but the answer to that question is still unknown. The same situation occurs with (D). It is proven that existence of a solution to (D) with $\alpha=\lambda_{1}$, the first Dirichlet eigenvalue (for which and only for which the eigenfunction is positive or negative on the whole $\Omega$ ), or a solution to (N) with $\alpha=\lambda_{2}$, both imply that $\Omega$ is a ball. Also, the existence of infinitely many eigenvalues to $(\mathrm{N})$ or $(\mathrm{D})$ in the above spaces implies that $\Omega$ is a metric ball (see $[\mathrm{Be}],[\mathrm{BeY}]$ ).

J. Serrin in 1971 (see [Se]) considered, in particular, the following overdetermined boundary value problem

$$
\begin{cases}\Delta u=-1, & \text { in } \Omega, \\ u=0, \frac{\partial u}{\partial n}=\mathrm{constant}, & \text { on } \partial \Omega .\end{cases}
$$

He proved that if $X=\mathbb{R}^{n}$ and (0.2) has a solution, then $\Omega$ must be a ball (another proof was given by H. F. Weinberger (see [Wei])). R. 
Molzon (see [Mo]) extended this result to the cases when $X$ is real hyperbolic space $\mathbb{H}^{n}$ or upper hemisphere $S_{+}^{n}$.

In this work we show the existence of a number of domains in different spaces for which (N) and (D) admit solutions for infinitely many eigenvalues; $(0.2)$ has a unique solution in each such a domain. These domains don't have to be metric balls and, moreover, in some cases, metric balls don't necessarily admit such solutions. Also, we consider some possible generalizations to the Schiffer Conjecture and other (essentially more complicated) overdetermined boundary value problems, including higher order equations, nonlinear equations, timedependent equations, and the spread of blow-up.

\section{Domains with homogeneous boundaries.}

Definition 2. Let $X$ be a Riemannian manifold, $\Gamma$ a regular $\left(C^{k}, C^{\infty}\right.$ or analytic) and connected hypersurface in $X . \Gamma$ is called a homogeneous hypersurface in $X$ if $\Gamma$ is an orbit of a subgroup $G \subset \operatorname{ISO}(X)$ where $\operatorname{ISO}(X)$ is the group of all isometries of $X$.

REMARK. Obviously, the principal curvatures (see [Carm]) of a homogeneous hypersurface are constant, but the converse statement is not always true. If $X$ is $\mathbb{R}^{n}$ or a non-compact irreducible symmetric space of the first rank (see [H1]) and $\Gamma$ is compact, then the fact that $\Gamma$ is homogeneous in $X$ means that $\Gamma$ is a metric sphere in $X$ so that this definition contains nothing new. If $X$ is a non-compact irreducible symmetric space of higher rank (see [H1]), then there are no compact homogeneous hypersurfaces, i.e. surfaces of codimension 1 in $X$ (in fact, even metric spheres are not homogeneous in $X$ ). However, if $X$ is a compact irreducible symmetric space of the first rank, then there are plenty of homogeneous hypersurfaces in $X$ including non-spheres as well as spheres (we will consider them later).

Lemma 1. Let $X$ be a complete Riemannian manifold and $\Omega$ a bounded domain in $X$ as in Section 0 . If $\partial \Omega$ is homogeneous in $X$, then the group $G$ in Definition 2 can be chosen in such a way that $G \subset \operatorname{ISO}_{0}(X)$ (identity component of $\operatorname{ISO}(X)), G$ is compact, connected and any $g \in$ $G$ maps $\Omega$ onto itself.

Proof. Let $\widetilde{G}$ be the group of all $g \in \operatorname{ISO}(X)$ mapping $\partial \Omega$ onto itself. 
Then, of course, $\partial \Omega$ is a compact orbit of $\widetilde{G}$, and $\widetilde{G}$ is compact as well (see $[\mathrm{H} 1]$ ). Show that there is a compact subgroup $G_{\Omega} \subset \widetilde{G}$ such that any $g \in G_{\Omega}$ maps $\Omega$ onto itself. Indeed, take any $g \in G$. Let $\Omega^{\prime}=g(\Omega)$. Then $\partial \Omega^{\prime}=g(\partial \Omega)$ so that $\partial \Omega^{\prime}=\partial \Omega$ which is connected and regular. Since $X \backslash \partial \Omega$ consists of two connected components, namely, $\Omega$ and the complement of the closure $\bar{\Omega}, g(\Omega)$ can be only $\Omega$ itself or $\bar{\Omega}^{c}$.

Now consider three possible cases:

Case 1. $X$ is non-compact. Then $\bar{\Omega}^{c}$ is unbounded and $g(\Omega)$ can never be $\bar{\Omega}^{c}$. Thus, for any $g \in \widetilde{G}$ we have $g(\Omega)=\Omega$ as required so that assume $G_{\Omega}=\widetilde{G}$.

Case 2. $X$ is compact but $\Omega$ and $\bar{\Omega}^{c}$ are non-isometric to each other. Then, again $g(\Omega)$ can not be $\bar{\Omega}^{c}$, i.e. we always have $g(\Omega)=\Omega$ and can take $G_{\Omega}=\widetilde{G}$.

Case 3. $X$ is compact, $\Omega$ and $\bar{\Omega}^{c}$ are isometric to each other. Define $G_{\Omega}$ as the group of all $g \in \operatorname{ISO}(X)$ mapping $\Omega$ onto itself $\left(G_{\Omega}\right.$ contains at least the identical isometry). Obviously, $G_{\Omega}$ is compact as well as $\widetilde{G}$. Moreover, $G_{\Omega}$ is a normal (with index 2) subgroup of $\widetilde{G}$, and $\widetilde{G} \backslash G_{\Omega}$ is the set of all $g \in \operatorname{ISO}(X)$ mapping $\Omega$ onto $\bar{\Omega}^{c}$ (or conversely).

Take any point $p \in \partial \Omega$. Consider the orbit $S$ of $p$ under the action of $G_{\Omega}, S$ is a closed subset of $\partial \Omega$ (in the topology induced on $\partial \Omega$ ). Show that $S=\partial \Omega$. Indeed, suppose the contrary. Take any $q \in \partial \Omega \backslash S$. Then $\partial \Omega \backslash S$ is the orbit of $q$ under the action of $G_{\Omega}$ so that $\partial \Omega \backslash S$ is a closed subset of $\partial \Omega$. This implies that $S$ is an open subset of $\partial \Omega$ while $S$ is already closed in $\partial \Omega$. Since $\partial \Omega$ is connected, $S$ can be only $\varnothing$ or $\partial \Omega$. But $S \ni p$ is non-empty so that $S=\partial \Omega$. Therefore, $\partial \Omega$ is the orbit of $G_{\Omega}$.

Thus, we can always choose $G_{\Omega}$ as a compact subgroup of $\operatorname{ISO}(X)$ (and, of course, of $\widetilde{G}$ ) such that any $g \in G_{\Omega}$ maps $\Omega$ onto itself. Since $G_{\Omega}$ is compact, it contains finitely many connected components. Let $G$ be the identity component, $G$ is a compact subgroup of $G_{\Omega}$. Prove that $\partial \Omega$ is the orbit of $G$. If $G_{\Omega}$ has only one component, i.e. $G$ itself, the claim is obvious. If there are more than one components, take any point $p \in \partial \Omega$ and consider the orbit $S_{1}$ of $p$ under the action of $G, S_{1}$ is the closed subset of $\partial \Omega$. Show that $S_{1}=\partial \Omega$. Suppose not. Since $G$ is acting on $\partial \Omega, \partial \Omega$ is split into finitely many orbits of $G$, call them $S_{1}, S_{2}, \ldots, S_{k}, k \geq 2$. All they are isometric to one another so that $\partial \Omega$ is split into a finite number $(k \geq 2)$ of closed non-empty sets. But this contradicts the connectivity of $\partial \Omega$. 
Therefore, $\partial \Omega$ is the orbit of $G$ and $G$ is chosen as required. The proof of Lemma 1 is completed.

Below we always assume that $G$ is chosen as described in Lemma 1.

As explained in Introduction, we can assume that $\partial \Omega$ is $C^{\infty}$ (even real-analytic) which implies that classical (not overdetermined) Neumann and Dirichlet eigenfunctions are at least $C^{2}$ (in fact, $C^{\infty}$ as well) (see $[R])$.

Lemma 2. Let $\Omega$ be a bounded domain in $X$ as in Section 0 . Then the classical Neumann Problem (respectively, the classical Dirichlet Problem) for $\Omega$ has infinitely many eigenvalues $\alpha>0$ with corresponding eigenfunctions $f_{\alpha}(x)$ satisfying the condition

$$
\int_{\partial \Omega} f_{\alpha}(x) d s \neq 0
$$

or, respectively,

$$
\int_{\partial \Omega} \frac{\partial f_{\alpha}(x)}{\partial n} d s \neq 0
$$

The latter condition (for classical Dirichlet Problem) is equivalent to

$$
\int_{\Omega} f_{\alpha}(x) d x \neq 0
$$

Proof. The equivalence of (1.2) and (1.3) is obvious because of Gauss Theorem:

$$
\int_{\partial \Omega} \frac{\partial f_{\alpha}(x)}{\partial n} d s=\int_{\Omega} \Delta f_{\alpha}(x) d x=-\alpha \int_{\Omega} f_{\alpha}(x) d x .
$$

Now, take any real $\lambda$ which is not an eigenvalue for classical Neumann Problem (respectively, classical Dirichlet Problem). Then usual boundary value problem

$$
\begin{cases}\Delta u+\lambda u=0, & \text { in } \Omega, \\ \frac{\partial u}{\partial n}=1, & \text { on } \partial \Omega .\end{cases}
$$


or, respectively,

$$
\begin{cases}\Delta u+\lambda u=0, & \text { in } \Omega, \\ u=1, & \text { on } \partial \Omega .\end{cases}
$$

has a unique regular solution, call it $\varphi(x)$. For Dirichlet Problem (1.5) one can assume $\lambda=0$ so that $\varphi(x) \equiv 1$. Then, if $f_{\alpha}(x)$ is an eigenfunction for Neumann Problem, we can write

$$
f_{\alpha}(x)=f_{\alpha}(x) \frac{\partial \varphi(x)}{\partial n}-\frac{\partial f_{\alpha}(x)}{\partial n} \varphi(x), \quad \text { on } \partial \Omega,
$$

and if $f_{\alpha}(x)$ is an eigenfunction for Dirichlet Problem, we have

$$
\frac{\partial f_{\alpha}(x)}{\partial n}=\frac{\partial f_{\alpha}(x)}{\partial n} \varphi(x)-f_{\alpha}(x) \frac{\partial \varphi(x)}{\partial n}, \quad \text { on } \partial \Omega .
$$

In both cases, Gauss' Theorem implies

$$
\begin{aligned}
\int_{\partial \Omega}\left(f_{\alpha}(x) \frac{\partial \varphi(x)}{\partial n}\right. & \left.-\frac{\partial f_{\alpha}(x)}{\partial n} \varphi(x)\right) d s \\
& =\int_{\partial \Omega}\left(f_{\alpha}(x) \nabla \varphi(x)-\left(\nabla f_{\alpha}(x)\right) \varphi(x)\right) \cdot \vec{n} d s \\
& =\int_{\Omega} \nabla \cdot\left(f_{\alpha}(x) \nabla \varphi(x)-\left(\nabla f_{\alpha}(x)\right) \varphi(x)\right) d x \\
& =\int_{\Omega}\left(\nabla f_{\alpha}(x) \cdot \nabla \varphi(x)+f_{\alpha}(x) \Delta \varphi(x)\right. \\
& =\int_{\Omega}\left(f_{\alpha}(x) \Delta \varphi(x)-\Delta f_{\alpha}(x) \cdot \varphi(x)\right) d x \\
& =\int_{\Omega}\left(f_{\alpha}(x)(-\lambda \varphi(x))+\alpha f_{\alpha}(x) \varphi(x)\right) d x \\
& =(\alpha-\lambda) \int_{\Omega} f_{\alpha}(x) \varphi(x) d x .
\end{aligned}
$$

Since $\lambda$ is not an eigenvalue, it can not equal $\alpha$ so that the condition (1.1) for Neumann Problem or, respectively, (1.2) for Dirichlet Problem is equivalent to

$$
\int_{\Omega} f_{\alpha}(x) \varphi(x) d x \neq 0 .
$$


Neumann Problem (respectively, Dirichlet Problem) has an increasing sequence of eigenvalues approaching to infinity

$$
\alpha_{1}<\alpha_{2}<\cdots<\alpha_{k}<\cdots
$$

where $\alpha_{1}=0$ (respectively, $\alpha_{1}>0$ ) and the multiplicity of $\alpha_{1}$ (i.e., the dimension of corresponding eigenspace) is 1 . Then $L^{2}(\Omega)$ can be decomposed into the direct sum of mutually orthogonal eigenspaces $E\left(\alpha_{k}\right), k \geq 1$

$$
L^{2}(\Omega)=E\left(\alpha_{1}\right) \oplus E\left(\alpha_{2}\right) \oplus \cdots \oplus E\left(\alpha_{k}\right) \oplus \cdots
$$

Now suppose that the statement of Lemma 2 is not true, i.e. the integral in (1.6) is non-zero for at most finitely many eigenvalues, call them $\alpha_{k_{1}}, \alpha_{k_{2}}, \ldots, \alpha_{k_{l}}$. Then $\varphi(x)$ is orthogonal to all $E\left(\alpha_{k}\right), k \geq 1$ in $L^{2}(\Omega)$, decomposition (1.7), except, maybe, $E\left(\alpha_{k_{1}}\right), E\left(\alpha_{k_{2}}\right), \ldots, E\left(\alpha_{k_{l}}\right)$ (at least one such $E\left(\alpha_{k_{i}}\right), i \geq 1$ exists since otherwise $\left.\varphi(x) \equiv 0\right)$. So $\varphi(x)$ must be in the finite direct sum of these eigenspaces, i.e.

$$
\varphi(x) \in E\left(\alpha_{k_{1}}\right) \oplus E\left(\alpha_{k_{2}}\right) \oplus \cdots \oplus E\left(\alpha_{k_{l}}\right)
$$

or

$$
\begin{aligned}
& \varphi(x)=\varphi_{1}(x)+\varphi_{2}(x)+\cdots+\varphi_{l}(x), \\
& \varphi_{i}(x) \in E\left(\alpha_{k_{i}}\right), \quad 1 \leq i \leq l,
\end{aligned}
$$

where $\varphi_{i}(x)$ are eigenfunctions for $\alpha_{k_{i}}$ (or, some of them may be zero). Now consider the two boundary value problems separately.

Case 1. For the Neumann Problem

$$
\frac{\partial \varphi}{\partial n}=\frac{\partial \varphi_{1}}{\partial n}+\frac{\partial \varphi_{2}}{\partial n}+\cdots+\frac{\partial \varphi_{l}}{\partial n}=0+0+\cdots+0=0, \quad \text { on } \partial \Omega,
$$

while $\partial \varphi / \partial n=1$ on $\partial \Omega$ by (1.4). This contradiction proves the statement of Lemma 2 for the Neumann Problem.

Case 2. For the Dirichlet Problem

$\varphi(x)=\varphi_{1}(x)+\varphi_{2}(x)+\cdots+\varphi_{l}(x)=0+0+\cdots+0=0, \quad$ on $\partial \Omega$,

while $\varphi(x)=1$ on $\partial \Omega$ by (1.5). This contradiction proves the statement of Lemma 2. for the Dirichlet Problem. 
Since we have considered both Neumann and Dirichlet Problems, the proof of Lemma 2 is completed.

Theorem 1. Let $X$ be Riemannian manifold and $\Omega$ a bounded domain in $X$ as in the Introduction. Suppose $\partial \Omega$ is homogeneous in $X$. Let $\alpha>0$ be an eigenvalue for the classical Neumann Problem (respectively, classical Dirichlet Problem) on $\Omega$. Then, the Overdetermined Neumann Problem (N) (respectively, Overdetermined Dirichlet Problem (D)) admits a solution for $\alpha$ if and only if $\alpha$ has an eigenfunction $f_{\alpha}(x)$ for the classical Neumann (respectively, Dirichlet) Problem satisfying the condition (1.1) (respectively, (1.2) if and only if (1.3)). In particular, any eigenfunction for the first Dirichlet eigenvalue (in the classical problem) is a solution for $(\mathrm{D})$.

Proof. If (N) (respectively, (D)) has a solution for some eigenvalue $\alpha>0$, then this solution obviously satisfies (1.1) (respectively, (1.2)).

Now we have to prove the other direction. Let $f_{\alpha}(x)$ be an eigenfunction corresponding to an eigenvalue $\alpha>0$ for classical Neumann (respectively, Dirichlet) Problem and satisfying (1.1) (respectively, (1.2)). Take any $g \in G$. Since the Laplace-Beltrami operator is invariant under isometries (see [H1]), i.e. $\Delta\left(f_{\alpha} \circ g\right)=\left(\Delta f_{\alpha}\right) \circ g$, the composition $f_{\alpha} \circ g$ satisfies the equation

$$
\Delta\left(f_{\alpha} \circ g\right)+\alpha\left(f_{\alpha} \circ g\right)=0 .
$$

On the other hand, $G$ acts transitively on $\partial \Omega$ and any isometry is a conformal map so that

$$
\frac{\partial\left(f_{\alpha} \circ g\right)}{\partial n}=\left(\frac{\partial f_{\alpha}}{\partial n}\right) \circ g, \quad \text { on } \partial \Omega .
$$

In the case of Neumann Problem, (1.10) implies that $\partial\left(f_{\alpha} \circ g\right) / \partial n$ vanishes on $\partial \Omega$ as well as $\partial f_{\alpha} / \partial n$. In the case of Dirichlet Problem, $\left(f_{\alpha} \circ g\right)$ vanishes on $\partial \Omega$ as well as $f_{\alpha}$. Therefore, in both cases $f_{\alpha} \circ g$ is also an eigenfunction for the eigenvalue $\alpha$ and this is true for any $g \in G$.

Now, since $G$ is a compact Lie group, it has Haar measure $\mu$ which is unique up to the assumption that $\mu(G)=1$. Given any function $\psi(x)$ continuous on $\partial \Omega$ and any point $p \in \partial \Omega$, invariance always implies that the average value is

$$
\int_{G} \psi(g \cdot p) d g=\frac{1}{A_{0}} \int_{\partial \Omega} \psi(x) d s,
$$


where $g \cdot p$ means $g(p)$ and $A_{0}$ is the area of $\partial \Omega$.

Now define a new function $f(x)$ on $\bar{\Omega}$ (in fact, in some neighborhood of $\bar{\Omega}$ ) in the following way

$$
f(x)=\int_{G} f_{\alpha} \circ g(x) d g=\int_{G} f_{\alpha}(g \cdot x) d g .
$$

Since the Haar measure on $G$ is invariant under the action of $G$, so is $f(x)$, i.e.

$$
f \circ h(x) \equiv f(x), \quad \text { for any } h \in G .
$$

Because of (1.9) we have

$$
\begin{aligned}
\Delta f(x) & =\Delta\left(\int_{G} f_{\alpha} \circ g(x) d g\right) \\
& =\int_{G} \Delta\left(f_{\alpha} \circ g(x)\right) d g \\
& =\int_{G}\left(-\alpha f_{\alpha} \circ g(x)\right) d g \\
& =-\alpha \int_{G} f_{\alpha} \circ g(x) d g \\
& =-\alpha f(x),
\end{aligned}
$$

i.e. $f(x)$ satisfies

$$
\Delta f+\alpha f=0 .
$$

Since $G$ acts transitively on $\partial \Omega,(1.13)$ implies that $f(x)$ is constant on $\partial \Omega$. Moreover, in view of $(1.11), f(x)$ on $\partial \Omega$ can be computed as

$$
f(x)=\int_{G} f_{\alpha} \circ g(x) d g=\frac{1}{A_{0}} \int_{y \in \partial \Omega} f_{\alpha}(y) d s, \quad x \in \partial \Omega .
$$

Similarly, because of (1.10) we have

$$
\frac{\partial f(x)}{\partial n}=\frac{\partial}{\partial n} \int_{G} f_{\alpha} \circ g(x) d g=\int_{G} \frac{\partial\left(f_{\alpha} \circ g(x)\right)}{\partial n} d g=\int_{G}\left(\frac{\partial f_{\alpha}}{\partial n}\right) \circ g(x) d g,
$$

so that we have

$$
\frac{\partial f(x)}{\partial n}=\frac{1}{A_{0}} \int_{y \in \partial \Omega} \frac{\partial f_{\alpha}}{\partial n}(y) d s, \quad x \in \partial \Omega,
$$


and $\partial f / \partial n$ is constant on $\partial \Omega$ as well as $f(x)$.

Now we are close to the end. According to (1.14), we have $\Delta f+$ $\alpha f=0$ on $\partial \Omega$. Again, consider both problems separately.

Case 1. For the Neumann Problem, $\partial f_{\alpha} / \partial n$ vanishes on $\partial \Omega$ and, because of (1.16), so does $\partial f / \partial n$. Also, (1.15) implies that $f(x)$ is some non-zero constant on $\partial \Omega$ (by the condition (1.1)). Therefore, $f(x)$ is a solution to Overdetermined Neumann Problem (N).

Case 2. For the Dirichlet Problem, $f_{\alpha}(x)$ vanishes on $\partial \Omega$ and, because of (1.15), so does $f(x)$. Also, (1.16) implies that $\partial f / \partial n$ is some non-zero constant on $\partial \Omega$ (by the condition (1.2)). Therefore, $f(x)$ is a solution to Overdetermined Dirichlet Problem (D).

In particular, if $\alpha$ is the first Dirichlet eigenvalue, any corresponding eigenfunction $f_{\alpha}(x)$ has a constant sign on $\Omega$ (for non-zero values) and the condition (1.2) is satisfied. Then $f(x)$ is a solution to (D). In fact, $f=f_{\alpha}$ in this case because all eigenfunctions for $\alpha$ coincide up to a constant multiple and (1.15) implies that $f(x)=f_{\alpha}(x)$ on $\partial \Omega$.

Since we have considered both Neumann and Dirichlet Problems, the proof of Theorem 1 is completed.

REMARK. Using the same technique as in Theorem 1, we can prove that $(0.2)$ has a unique solution in $\Omega$ whenever $\partial \Omega$ is homogeneous in $X$.

Applying Lemma 2, we get:

Corollary 1. Let $X$ be Riemannian manifold and $\Omega$ a bounded domain in $X$ as in Section 0 . If $\partial \Omega$ is homogeneous in $X$, then $(\mathrm{N})$ (respectively, (D)) admits solutions (overdetermined eigenfunctions) $f_{i}(x)$ for infinitely many eigenvalues $\alpha_{i}$. These eigenfunctions, $f_{i}(x)$ (taken once for each $\alpha_{i}$ and with $\alpha_{1}=0, f_{1}(x)$ a constant for $(\mathrm{N})$ ), form an orthogonal basis for the subspace of all $G$-invariant functions in $L^{2}(\Omega)$, i.e. for

$$
\begin{aligned}
L_{G}^{2}(\Omega)=\left\{f \in L^{2}(\Omega):\right. & \text { for all } g \in G \\
& \text { such that } \left.f \circ g=f \text { in } L^{2}(\Omega)\right\} .
\end{aligned}
$$

REMARK. We can not automatically say that metric balls in any Riemannian manifold $X$ admit solutions to (N) or (D). One can guarantee that only if metric spheres in $X$ are homogeneous. This is, of course, 
true in $\mathbb{R}^{n}$ or irreducible symmetric spaces of the first rank but not in the spaces of higher rank or non-symmetric spaces (we will see examples below).

Generalized Schiffer Conjecture (GSC). The natural generalization of Schiffer Conjecture to an arbitrary Riemannian manifold $X$ is that the existence of a solution to $(\mathrm{N})$ implies that $\partial \Omega$ is homogeneous in $X$. In $\mathbb{R}^{n}$ or non-compact irreducible symmetric spaces of the first rank (GSC) and (SC) are equivalent since there is no difference between metric spheres and compact homogeneous hypersurfaces in these spaces (and the answer is still unknown). The examples below show that this is not so in general. The same question, of course, applies to (D) (as well as to (0.2)). Also, it is interesting to consider a weaker version of the problem: if $(\mathrm{N})$ or $(\mathrm{D})$ admits solutions for infinitely many eigenvalues, does it imply that $\partial \Omega$ is homogeneous in $X$ ? (As noted above, in $\mathbb{R}^{n}$ or non-compact irreducible symmetric spaces of the first rank this is true).

In next sections we will see a number of different spaces where domains with homogeneous as well as with non-homogeneous boundaries admit solutions to (N) and (D) for infinitely many eigenvalues.

\section{Domains with isoparametric boundaries in symmetric spaces.}

In this section we will construct a number of specific domains $\Omega$ admitting solutions to (N) and (D) in the classical compact irreducible symmetric spaces of the first rank, namely, $S^{n}, R P^{n}, C P^{n}, H P^{n}$, see [H2] (we don't consider the exceptional case $P^{16}$ (Cay)).

ExAmple 1. Suppose $X=S^{n} \subset \mathbb{R}^{n+1}=\mathbb{R}^{k} \times \mathbb{R}^{l}, n=k+l-1$. Let

$$
f_{k, l}(x)=l\left(x_{1}^{2}+\cdots+x_{k}^{2}\right)-k\left(x_{k+1}^{2}+\cdots+x_{k+l}^{2}\right) .
$$

Let $\Omega_{k, l}=\left\{x \in S^{n}: f_{k, l}(x)>0\right\}$ so that $\partial \Omega_{k, l}=\left\{x \in S^{n}: f_{k, l}(x)=\right.$ $0\}$. Then $\partial \Omega_{k, l}=S^{k-1}\left(r_{1}\right) \times S^{l-1}\left(r_{2}\right)$ (with $r_{1}=\sqrt{k /(k+l)}, r_{2}=$ $\sqrt{l /(k+l)})$ which is called generalized Clifford torus. It is connected if both $k, l>1$; if either $k$ or $l$ equals $1, \partial \Omega_{k, l}$ consists of two components, isometric to $S^{n-1}(\sqrt{n /(n+1)})$. Obviously, for $k, l \geq 2, \partial \Omega_{k, l}$ admits a transitive isometry group, namely, $S O(k) \times S O(l)$. Therefore, Theorem 1 implies that (D) has a solution in $\Omega_{k, l}$. This conclusion (for that 
particular domain) was obtained by M. Karlovitz in 1990 (see [Kar]) by direct computations. He showed that $f_{k, l}(x)$ is such a solution itself (in fact, it is first eigenfunction mentioned in Theorem 1).

Indeed, the second degree polynomial $f_{k, l}(x)$ is harmonic on $\mathbb{R}^{n+1}$. Then since $f_{k, l}(x)$ is also homogeneous it is known that $f_{k, l}$ restricted to $S^{n}$ is a solution to the global eigenvalue problem

$$
\Delta_{S} u+\alpha u=0, \quad \text { on } S^{n},
$$

for $\alpha=2(k+l)$ where $\Delta_{S}$ is the Laplace-Beltrami operator on $S^{n}$ (see $[\mathrm{Ch}])$. After computations we will have

$$
\left\|\nabla_{S} f_{k, l}\right\|^{2}=4\left(l-f_{k, l}\right)\left(k+f_{k, l}\right),
$$

where $\nabla_{S}$ is the gradient along $S^{n}$, so that $\left\|\nabla_{S} f_{k, l}\right\|=2 \sqrt{k l}$ on $\partial \Omega_{k, l}$ (where $f_{k, l}=0$ ). Now because $\partial \Omega$ is a level surface of $f_{k, l}$, a normal (outward) vector to it will be $-\nabla_{S} f_{k, l} /\left\|\nabla_{S} f_{k, l}\right\|$. Then

$$
\frac{\partial_{S} f_{k, l}}{\partial n}=-\frac{\nabla_{S} f_{k, l}}{\left\|\nabla_{S} f_{k, l}\right\|} \cdot \nabla_{S} f_{k, l}=-\left\|\nabla_{S} f_{k, l}\right\|=-2 \sqrt{k l}, \text { on } \partial \Omega_{k, l} .
$$

Therefore, $f_{k, l}(x)$ is a solution for (D) on $\Omega_{k, l}$ (it is a first eigenfunction since $f_{k, l}(x) \geq 0$ on $\left.\Omega_{k, l}\right)$. Of course, this is not the only solution since Theorem 1 implies that both (N) and (D) have solutions for infinitely many eigenvalues (and problem (0.2) has a unique solution in $\Omega_{k, l}$ ).

In the above example, one can make the following very important

Observation. $\Delta_{S} f_{k, l}$ and $\left\|\nabla_{S} f_{k, l}\right\|^{2}$ can be expressed in terms of $f_{k, l}$ only.

The class of functions having this property is very interesting as well as the class of their level surfaces. Now we need a brief account of the theory of this subject in general case.

\subsection{Basic theory of isoparametric functions and hypersur- faces.}

Definition 3. Let $X$ be a Riemannian manifold and $f: X \longrightarrow R$ a smooth function on $X$ ( or on some open connected subset $U \subset X$ ). We 
say that $f$ is an isoparametric function on $X$ (or on $U \subset X$ ) if there exist smooth functions $A$ and $B$ defined on the image, $f(X) \subseteq R$, of $X$ under $f($ or $f(U) \subseteq R$, of $U$ under $f$ ) so that

$$
\begin{gathered}
\Delta f=A \circ f, \\
\|\nabla f\|^{2}=B \circ f .
\end{gathered}
$$

Regular level surfaces of $f$ are called isoparametric hypersurfaces in $X$.

A function $f$ satisfying (2.3) is called transnormal. Geometrical meaning of transnormality of $f$ is that level sets of $f$ are parallel (see [Q1]). That is, for any two level sets of $f$, say, $P \subset X, Q \subset X$ and given any two points $x \in P, y \in Q$, we have dist $(x, Q)=\operatorname{dist}(y, P)$.

By the definition, any isoparametric function is automatically transnormal and, for any regular level surface of $f$, call it $M \subset X$, its mean curvature

$$
\chi=-\frac{1}{n-1} \operatorname{div}\left(\frac{\nabla f}{\|\nabla f\|}\right)
$$

is constant along $M$ (see [Q1]). Thus, $f$ is isoparametric if all its regular level surfaces are parallel and have constant mean curvatures (generally speaking, different for different levels).

Now, for a regular hypersurface $M \subset X$ to be isoparametric means that any hypersurface $N$, sufficiently close and parallel to $M$, has constant mean curvature (see [Q1]). That is, the mean curvature depends only on $N$ but not on a position on $N$ (including $N=M$ ). Therefore, the concept of isoparametricity of hypersurface is geometrical and doesn't depend on defining isoparametric function (such a function can't be unique).

It is important that the definitions and geometrical interpretation of isoparametricity can be given for arbitrary Riemannian manifold. For the first time the above notions were introduced and intensively studied by E. Cartan (see [Cart]) in the spaces of constant sectional curvature. He proved the following beautiful result.

Theorem (E. Cartan, 1938). In a space $X$ of constant sectional curvature (basically, $X$ is $\mathbb{R}^{n}$, sphere $S^{n}$ or hyperbolic space $\mathbb{H}^{n}$ ), a hypersurface $M$ is isoparametric in $X$ if and only if all principal curvatures are constant. If $X$ is $\mathbb{R}^{n}$ or $\mathbb{H}^{n}$ and $M$ is a compact hypersurface, then $M$ is isoparametric in $X$ if and only if $M$ is a metric sphere. 
Since any homogeneous hypersurface has constant principal curvatures, in any space $X$ of constant sectional curvature one has the following:

Proposition 1. If a hypersurface $M$ is homogeneous in $X$, then $M$ is isoparametric in $X$.

REMARK. In fact, Proposition 1 holds in any Riemannian manifold $X$. If $X$ is $\mathbb{R}^{n}$ or a non-compact irreducible symmetric space of the first rank, then the class of known isoparametric compact hypersurfaces coincides with that of the homogeneous compact hypersurfaces, i.e. metric spheres.

Cartan classified isoparametric hypersurfaces in $S^{n}, n=d+1$ with $g$ distinct principal curvatures for $g=1,2,3$ :

Case $g=1$. Subsphere $S^{n-1}(r)$ in $S^{n}$.

Case $g=2$. Generalized Clifford Torus $S^{k-1}\left(r_{1}\right) \times S^{l-1}\left(r_{2}\right)$ in $S^{n}$ (Example 1).

Case $g=3 . \quad M^{d} \subset S^{d+1} \subset \mathbb{R}^{d+2}, d=3,6,12,24, M^{d}$ is a level surface of the following function $f$ on $\mathbb{R}^{d+2}$ (whose restriction on $S^{d+1}$ is isoparametric)

$$
\begin{aligned}
f(x)= & x_{5}^{3}+\frac{3}{2} x_{5}\left(\left|z_{1}\right|^{2}+\left|z_{3}\right|^{2}-2\left|z_{2}\right|^{2}-2 x_{4}^{2}\right) \\
& +\frac{3 \sqrt{3}}{2} x_{4}\left(\left|z_{1}\right|^{2}-\left|z_{3}\right|^{2}\right)-\frac{3 \sqrt{3}}{2}\left(z_{1} z_{2} z_{3}+\bar{z}_{3} \bar{z}_{2} \bar{z}_{3}\right),
\end{aligned}
$$

where $z_{1}, z_{2}, z_{3}$ are real, complex or quaternions or octonians (according to $d=3,6,12,24), x_{4}, x_{5}$ are always real.

All hypersurfaces in the above Cartan's examples are homogeneous. Cartan proposed the following question: If $M$ is an isoparametric surface in $S^{n}$, is $M$ necessarily homogeneous in $S^{n}$ ?

The negative answer to that question was obtained by H. Ozeki and M. Takeuchi in 1975 (see [OzT]) for $g=4$. They gave a number of different examples but we will show only two of them, for $n=15$ and $n=31$. Let $F$ be the quaternion algebra (respectively, non-associative octonian algebra). The dimension of $F$ over $\mathbb{R}$ is 4 (respectively, 8) so that $\mathbb{R}^{n+1}$ can be written as

$$
\mathbb{R}^{n+1}=F^{2} \times F^{2}=F \times F \times F \times F
$$


(as a Euclidean space). Let

$$
u=\left(\begin{array}{l}
u_{0} \\
u_{1}
\end{array}\right) \in F^{2}, \quad v=\left(\begin{array}{l}
v_{0} \\
v_{1}
\end{array}\right) \in F^{2}, \quad \text { all } u_{0}, u_{1}, v_{0}, v_{1} \in F .
$$

Define the scalar product in $F$ as

$$
\left(q_{1}, q_{2}\right)=\frac{1}{2}\left(q_{1} \bar{q}_{2}+q_{2} \bar{q}_{1}\right), \quad q_{1}, q_{2} \in F,
$$

where $\bar{q}$ means conjugate to $q \in F$. In fact, this product coincides with the usual dot product in $F=\mathbb{R}^{4}$ (respectively, $F=\mathbb{R}^{8}$ ). Then the norm in $F$ is given by

$$
\|q\|=(q, q)^{1 / 2}, \quad q \in F .
$$

The scalar product and norm can be extended to $F^{2}=\mathbb{R}^{8}$ (respectively, $\left.F^{2}=\mathbb{R}^{16}\right)$ as

$$
(u, v)=\left(u_{0}, v_{0}\right)+\left(u_{1}, v_{1}\right)=\frac{1}{2}\left(u^{\prime} \bar{v}+v^{\prime} \bar{u}\right), \quad\|u\|=(u, u)^{1 / 2},
$$

where ' means transpose. Now define a function $f$ isoparametric on $\mathbb{R}^{n+1}=F^{2} \times F^{2}$ in the following way

$$
\begin{aligned}
f(u \times v)= & \left(\|u\|^{2}+\|v\|^{2}\right)^{2}-2 f_{0}(u \times v), \\
f_{0}(u \times v)= & 4\left(\left\|u^{\prime} \bar{v}\right\|^{2}-(u, v)^{2}\right) \\
& +\left(\left\|u_{1}\right\|^{2}-\left\|v_{1}\right\|^{2}+2\left(u_{0}, v_{0}\right)\right)^{2}, \quad u, v \in F^{2} .
\end{aligned}
$$

The restriction of $f$ on $S^{n}$ is an isoparametric function, and its level surfaces turn out to be non-homogeneous isoparametric hypersurfaces in $S^{n}$. (If one takes $F$ to be the complex field, one can obtain a function isoparametric on $S^{7} \subset \mathbb{R}^{8}$ whose level surfaces are homogeneous isoparametric hypersurfaces in $S^{7}$ with $g=4$.)

The complete geometric classification of isoparametric hypersurfaces in $S^{n}$ is still unknown. In $1980 \mathrm{H}$. Munzer (see [Mun]), in his beautiful work, reduced this classification problem to a (difficult) algebraic problem. He obtained the following results.

Proposition 2. Number $g$ of distinct principal curvatures for an isoparametric hypersurface $M$ in $S^{n}$ can be only: $g=1,2,3,4,6$. 
Proposition 3. Let $\chi_{0}>\chi_{1}>\cdots>\chi_{g-1}$ be the distinct principal curvatures of $M$. Then their multiplicities $m_{i}, i=0,1, \ldots, g-1$ are repeated as $m_{0}, m_{1}, m_{0}, m_{1}, \ldots$ If $g=1,3,6$, then $m_{0}=m_{1}$, i.e. all $m_{i}$ are equal. We have $n-1=g\left(m_{0}+m_{1}\right) / 2$.

Proposition 4. An isoparametric hypersurface $M$ of $g$ distinct principal curvatures is a level surface of so called Cartan polynomial $f(x)$. By the definition, such a polynomial is a homogeneous polynomial of the degree $g$ defined on $\mathbb{R}^{n+1} \supset S^{n}$ and satisfying

$$
\left\{\begin{array}{l}
\Delta_{R} f=c|x|^{g-2} \\
\left\|\nabla_{R} f\right\|^{2}=g^{2}|x|^{2 g-2}
\end{array}\right.
$$

where $\Delta_{R}$ and $\nabla_{R}$ mean the usual operations on $\mathbb{R}^{n+1}, c=\left(m_{1}-\right.$ $\left.m_{0}\right) g^{2} / 2$ so that $c=0$ if $g=1,3,6$. Namely, the restriction $V=\left.f\right|_{S^{n}}$ is isoparametric on $S^{n}$, its range is $[-1,1]$ and $M=V^{-1}(t)$ for some $t \in(-1,1)$. Given $M$, the polynomial $f$ is uniquely defined (up to \pm if $c=0)$.

Conversely, let $f$ be a Cartan polynomial, i.e. homogeneous polynomial of degree $g$ satisfying (2.6) for some $c$ with $g(n-1)-c \neq 0$, $g(n-1)+c \neq 0$. Let $V=\left.f\right|_{S^{n}}$. Then $V\left(S^{n}\right)=[-1,1]$ and for any $t \in(-1,1), M_{t}=V^{-1}(t)$ is a regular connected isoparametric hypersurface with $g$ distinct principal curvatures, $m_{0}=(g(n-1)-c) / g^{2}$, $m_{1}=(g(n-1)+c) / g^{2}\left(M_{-1}\right.$ and $M_{1}$ are so-called focal sets $)$. The restriction $V$ is satisfying

$$
\left\{\begin{array}{l}
\Delta V=-g(g+n-1) V+c, \\
\|\nabla V\|^{2}=g^{2}\left(1-V^{2}\right),
\end{array}\right.
$$

where $\Delta=\Delta_{S}, \nabla=\nabla_{S}$.

The restriction of a Cartan polynomial $f(x)$ on $S^{n}$ can be expressed as

$$
V(x)=\cos \left(g \cdot \operatorname{dist}\left(x, M_{1}\right)\right),
$$

so that, given any two numbers $a, b$ with $-1 \leq a<b \leq 1$, the distance between the corresponding level surfaces (including, possibly, focal sets) is

$$
\operatorname{dist}\left(M_{a}, M_{b}\right)=\frac{1}{g}(\arccos a-\arccos b)
$$


Any regular isoparametric hypersurface $M_{t}$ divides $X=S^{n}$ into two parts: $\Omega_{t}=\Omega_{t}^{+}=\{x \in X: V(x)>t\}$ and $\Omega_{t}^{-}=\{x \in X: V(x)<t\}$. S. Helgason gave an effective technique of computation of the volumes of metric balls and areas of spheres in symmetric spaces (see [H2]). This technique can be modified and generalized to isoparametric hypersurfaces in Riemannian manifolds (see [Sh]). The following Lemma 3 is contained in (see $[\mathrm{Sh}]$ ) but we reproduce it here for the reader's convenience.

Lemma 3. The volume of $\Omega_{t}$ can be computed as

$$
\operatorname{Vol}\left(\Omega_{t}\right)=\beta \int_{t}^{1}(1-\tau)^{\left(m_{0}-1\right) / 2}(1+\tau)^{\left(m_{1}-1\right) / 2} d \tau,
$$

where $\beta>0$ is a constant (which can be determined from the conditions $\operatorname{Vol}\left(\Omega_{1}\right)=0$ and that $\operatorname{Vol}\left(\Omega_{-1}\right)$ is actually the volume of $X$, in this case, $\left.S^{n}\right)$.

ProOF. Integrate the first identity of (2.7) or, which is actually the same,

$$
\Delta V=g^{2}\left(\frac{m_{1}-m_{0}}{2}-\left(1+\frac{m_{1}+m_{0}}{2}\right) V\right)
$$

over $\Omega_{t}$. Using Gauss formula, we get

$$
\int_{\partial \Omega_{t}} \frac{\partial V}{\partial n} d s=g^{2} \int_{\Omega_{t}}\left(\frac{m_{1}-m_{0}}{2}-\left(1+\frac{m_{1}+m_{0}}{2}\right) V\right) d x
$$

Since

$$
\left.\frac{\partial V}{\partial n}\right|_{\partial \Omega_{t}}=\left.\nabla V \cdot \frac{-\nabla V}{\|\nabla V\|}\right|_{V=t}=\left.(-\|\nabla V\|)\right|_{V=t}=-g \sqrt{1-t^{2}},
$$

we have

$$
-A(t) \cdot g \sqrt{1-t^{2}}=g^{2} \int_{\Omega_{t}}\left(\frac{m_{1}-m_{0}}{2}-\left(1+\frac{m_{1}+m_{0}}{2}\right) V\right) d x
$$

where $A(t)$ means the area of $M_{t}=\partial \Omega_{t}$. Now, the derivative of the volume $\operatorname{Vol}(t) \equiv \operatorname{Vol}\left(\Omega_{t}\right)$ is

$$
\frac{d \operatorname{Vol}(t)}{d t}=-\frac{A(t)}{\left.(\|\nabla V\|)\right|_{V=t}}=-\frac{A(t)}{g \sqrt{1-t^{2}}} .
$$


Differentiating (2.11), we get

$$
\begin{aligned}
\frac{d}{d t}\left(-A(t) \cdot g \sqrt{1-t^{2}}\right) & =g^{2}\left(\frac{m_{1}-m_{0}}{2}-\left(1+\frac{m_{1}+m_{0}}{2}\right) t\right) \frac{d \operatorname{Vol}(t)}{d t} \\
& =g^{2}\left(\frac{m_{1}-m_{0}}{2}-\left(1+\frac{m_{1}+m_{0}}{2}\right) t\right) \frac{-A(t)}{g \sqrt{1-t^{2}}}
\end{aligned}
$$

so that

$$
\frac{d}{d t}\left(A(t) \sqrt{1-t^{2}}\right)=\left(\frac{m_{1}-m_{0}}{2}-\left(1+\frac{m_{1}+m_{0}}{2}\right) t\right) \frac{A(t)}{\sqrt{1-t^{2}}} .
$$

Integrating this ordinary differential equation we have

$$
A(t)=\widetilde{\beta}(1-t)^{m_{0} / 2}(1+t)^{m_{1} / 2},
$$

where $\widetilde{\beta}>0$ is a constant. Therefore, by (2.12), we get (2.20) with $\beta=\widetilde{\beta} / g$.

Examples of Cartan polynomials are (2.4) for $g=3$ and (2.5) for $g=4$. If some regular level surface of $V(x)$ in $S^{n}$ is homogeneous (respectively, non-homogeneous), then so are all other regular level surfaces of $V(x)$ in $S^{n}$. Munzer's theory admits generalization to the classical projective spaces, namely, $X=R P^{n}, C P^{n}, H P^{n}$. Let's identify $S^{1}$ with the group of the complex numbers with the absolute value 1 and $S^{3}$ with that of quaternions. As follows from the work of Simon Mullen (1994) (see [Mul]), isoparametricity is preserved under natural projection which implies

Proposition 5. Let $\pi$ be the natural projection

$$
\begin{aligned}
& \pi: S^{m} \longrightarrow X=S^{m} / K=R P^{n}, C P^{n} \text { or } H P^{n}, \\
& K=\{ \pm 1\}, S^{1} \text { or } S^{3}, m=n, 2 n+1 \text { or } 4 n+3 .
\end{aligned}
$$

Let $f$ be a Cartan polynomial on $R^{m+1} \supset S^{m}$ which is invariant under the action of $K$ (say, left multiplication) and whose restriction $\widetilde{V}=\left.f\right|_{S^{m}}$ satisfies (2.7) (with $m$ instead of $n$ ). Then $V=\widetilde{V} \circ \pi^{-1}$ is uniquely defined isoparametric function on $X$ satisfying the same equations (2.7) so that level surfaces of $V$ are isoparametric hypersurfaces in $X$. In other words, the projections of (invariant under $K$ ) 
isoparametric hypersurfaces in $S^{m}$ are isoparametric hypersurfaces in $X$.

Conversely, any isoparametric hypersurface $M \subset X(X$ is a space described in (2.14)) is a level surface of

$$
V=\widetilde{V} \circ \pi^{-1}, \quad \widetilde{V}=\left.f\right|_{S^{m}},
$$

where $V, \widetilde{V}$ satisfy (2.7) and $f$ is a Cartan polynomial on $\mathbb{R}^{m+1} \supset S^{m}$ invariant under $K$. In other words, isoparametric hypersurfaces in $X$ are the projections of (invariant under $K$ ) isoparametric hypersurfaces in $S^{m}$.

REMARK. Generally, in the classical projective spaces of non-constant sectional curvature $\left(C P^{n}, H P^{n}\right)$ isoparametric hypersurfaces no longer need to have constant principal curvatures (see [Q2]). However, this condition still holds for homogeneous hypersurfaces. Obviously, any Cartan polynomial $f$ of even degree $(g=2,4,6)$ on $\mathbb{R}^{n+1} \supset S^{n}$ is invariant under $K=\{ \pm 1\}$ so that $V$ is isoparametric on $R P^{n}$ (level surfaces of $V$ are homogeneous in $R P^{n}$ if and only if level surfaces of $\widetilde{V}$ are homogeneous in $S^{n}$ ). Moreover, there are many second degree Cartan polynomials on $\mathbb{R}^{m+1} \supset S^{m}$ invariant under $K=S^{1}, S^{3}$. They can be formed as

$$
f(x)=x_{1}^{2}+\cdots+x_{k}^{2}-x_{k+1}^{2}-\cdots-x_{k+l}^{2},
$$

where $m=k+l-1$, both $k$ and $l$ are divisible by 2 (when $K=$ $S^{1}$ ) or by 4 (when $K=S^{3}$ ). Cartan polynomials of fourth degree (on $R^{16} \supset S^{15}$ or on $R^{32} \supset S^{31}$ ) in (2.5) are also invariant under both $S^{1}$ and $S^{3}$ (only under $S^{1}$ on $R^{8} \supset S^{7}$, when $F$ is the complex field). Therefore, isoparametric hypersurfaces in the above projective spaces exist as well as isoparametric functions on them. All spaces $X=R P^{n}, C P^{n}, H P^{n}$ have homogeneous isoparametric hypersurfaces (not necessarily metric spheres) and each kind of these projective spaces has non-homogeneous isoparametric hypersurfaces for some dimensions. In particular, isoparametric hypersurfaces with non-constant principal curvatures in $X=C P^{n}, H P^{n}$ (see [Q2]) are always non-homogeneous.

It is important that Lemma 3 extends to the considered projective spaces $X$ (because the basic formulae don't change) (see [Sh]). 


\subsection{Solutions for $(\mathrm{N})$ and $(\mathrm{D})$.}

In general case, let $f(x)$ be an isoparametric function on any Riemannian manifold $X$, i.e. satisfying equations (2.2), (2.3). Given a smooth, real-valued function $h$ on $R$, for the composite function $h \circ f(x)=h(f(x))$ we can express

$$
\Delta h(f(x))=\|\nabla f\|^{2} h^{\prime \prime}(f)+\Delta f \cdot h^{\prime}(f)=B(f) h^{\prime \prime}(f)+A(f) h^{\prime}(f)
$$

and

$$
\|\nabla h(f(x))\|=\left|h^{\prime}(f)\right| \cdot\|\nabla f\|=\sqrt{B(f)} \cdot\left|h^{\prime}(f)\right| .
$$

Now we are ready to solve (N) and (D) for an arbitrary domain $\Omega \subset S^{n}$ with the boundary $\partial \Omega$ which is isoparametric in $S^{n}$. Indeed, if $\partial \Omega$ is homogeneous in $S^{n}$, Theorem 1 implies that both (N) and (D) have solutions for infinitely many eigenvalues. Since basic equations (2.7) for all the kinds of isoparametric hypersurfaces in sphere are virtually the same, $n-1=g\left(m_{0}+m_{1} / 2\right), c=g^{2}\left(m_{1}-m_{0} / 2\right)$ and (2.16), (2.17) yield

Theorem 2. Let $f(x)$ be a Cartan polynomial on $\mathbb{R}^{n+1} \supset S^{n}$ with the restriction $V=\left.f\right|_{S^{n}}$ satisfying $(2.7)$. Then, given $\varepsilon \in(-1,1)$, $\Omega=\left\{x \in S^{n}: V(x)>\varepsilon\right\}$ admits solutions to both (N) and (D) for infinitely many eigenvalues $\alpha>0$. These solutions have the form $u(x)=h(V(x))$ where $h(V)$ is a solution of hypergeometric equation

$$
\begin{aligned}
& \left(1-V^{2}\right) h^{\prime \prime}(V) \\
& +\left(\frac{m_{1}-m_{0}}{2}-\left(1+\frac{m_{1}+m_{0}}{2}\right) V\right) h^{\prime}(V)+\lambda h(V)=0
\end{aligned}
$$

with $\lambda=\alpha / g^{2}, h$ regular at $V=1$ and $h^{\prime}(\varepsilon)=0$ for $(\mathrm{N})$ or $h(\varepsilon)=0$ for (D). In particular, any eigenfunction for the first classical Dirichlet eigenvalue is a solution for (D). Also, if there is a solution $h(V)$ of (2.18) with $\lambda=k\left(k+\left(m_{1}+m_{0}\right) / 2\right)$ or, equivalently, $\alpha=g k(g k+n-1)$ for an integer $k \geq 1$, then $h(V)$ is (proportional to) a Jacobi polynomial of the degree $k$ so that corresponding overdetermined eigenfunction $u(x)$ can be extended to a global eigenfunction on $S^{n}$.

REMARK. Similarly, under the conditions of Theorem 2, (0.2) has a unique solution in the form $u(x)=h(V(x))$ where

$$
\left(1-V^{2}\right) h^{\prime \prime}(V)+\left(\frac{m_{1}-m_{0}}{2}-\left(1+\frac{m_{1}+m_{0}}{2}\right) V\right) h^{\prime}(V)=-\frac{1}{g^{2}}
$$


with $h$ regular at $V=1$ and $h(\varepsilon)=0$.

Using Proposition 5, we can extend these results to the projective spaces.

Theorem 3. Let $X=S^{m} / K$ be one of the projective spaces described in Proposition $5(\operatorname{dim} X=n)$. Let $f$ be a Cartan polynomial on $\mathbb{R}^{m+1} \supset$ $S^{m}$ which is invariant under $K$ and whose restriction $\widetilde{V}=\left.f\right|_{S^{m}}$ satisfies (2.7) (with $m$ instead of $n$ ) as well as $V=\widetilde{V} \circ \pi^{-1}$. Then, given $\varepsilon \in(-1,1), \Omega=\{x \in X: V(x)>\varepsilon\}$ admits solutions to both $(\mathrm{N})$ and (D) for infinitely many eigenvalues $\alpha>0$. These solutions have the form $u(x)=h(V(x))$ where $h(V)$ is a solution of hypergeometric equation (2.18) with the same conditions as in Theorem 2 . In particular, any eigenfunction for the first classical Dirichlet eigenvalue is a solution for (D). Also, if there is a solution $h(V)$ of (2.18) with $\lambda=k(k+$ $\left.\left(m_{1}+m_{0}\right) / 2\right)$ or, equivalently, $\alpha=g k(g k+m-1)$ for an integer $k \geq 1$, then $h(V)$ is (proportional to) a Jacobi polynomial of the degree $k$ so that corresponding overdetermined eigenfunction $u(x)$ can be extended to a global eigenfunction on $X$.

REMARK. Given $\varepsilon \in(-1,1)$, the eigenfunctions $h_{i}(V)$ (taken once for each eigenvalue $\lambda_{i}=\alpha_{i} / g^{2}$ and with $\lambda_{1}=0, h_{1}(V)$ a constant for $(\mathrm{N})$ ) of the hypergeometric equation (2.18) (for either $(\mathrm{N}), h^{\prime}(\varepsilon)=0$ or (D), $h(\varepsilon)=0$ ) form an orthogonal basis in a weighted one variable functional space $L_{w}^{2}[\varepsilon, 1]$ with the weight

$$
w(V)=(1-V)^{\left(m_{0}-1\right) / 2}(1+V)^{\left(m_{1}-1\right) / 2} .
$$

The corresponding eigenfunctions $u_{i}(x)=h_{i}(V(x))$ are orthogonal to one another in $L^{2}(\Omega)$. This correspondence has a much deeper meaning. Indeed, given two functions $p(V), q(V)$ on $[\varepsilon, 1]$, we automatically get two functions $p(V(x)), q(V(x))$ on $\bar{\Omega}$. Their product in $L^{2}(\Omega)$ can be written as

$$
\begin{aligned}
\langle p(V), q(V)\rangle_{L^{2}(\Omega)} & =\int_{V(x)>\varepsilon} p(V(x)) q(V(x)) d x \\
& =-\int_{t=\varepsilon}^{t=1} p(t) q(t) d \operatorname{Vol}\left(\Omega_{t}\right)
\end{aligned}
$$

where $d \operatorname{Vol}\left(\Omega_{t}\right)$ is an element of the volume between two parallel and close isoparametric hypersurfaces (level surfaces of $V$ ). Due to Lemma 
3 , the scalar product can be expressed as

$$
\begin{aligned}
\langle p(V), q(V)\rangle_{L^{2}(\Omega)} & =\beta \int_{\varepsilon}^{1} p(t) q(t)(1-t)^{\left(m_{0}-1\right) / 2}(1+t)^{\left(m_{1}-1\right) / 2} d t \\
& =\beta\langle p, q\rangle_{L_{w}^{2}[\varepsilon, 1]},
\end{aligned}
$$

where $\beta>0$ is a constant (not depending on $p, q, \varepsilon$ ). That is, the dot product (and the therefore the norm) of functions of the form $\varphi(V)$ in $L^{2}(\Omega)$ is equivalent to that in $L_{w}^{2}[\varepsilon, 1]$ and, very important, is actually the same (up to a constant multiple) for all kinds of isoparametric hypersurfaces and the considered spaces $X$ (depending only on $m_{0}$, $\left.m_{1}\right)$.

Similarly to $L_{G}^{2}(\Omega)$ in $(1.17)$, define the subspace

$$
\begin{aligned}
L_{V}^{2}(\Omega)=\left\{u \in L^{2}(\Omega):\right. & u=\varphi \circ V \text { in } L^{2}(\Omega), \\
& \varphi \text { is a function on }[\varepsilon, 1]\} .
\end{aligned}
$$

The meaning of (2.22) is that if $u \in L_{V}^{2}(\Omega)$ is continuous, then it is constant along any level surface of $V(x)$ in $\Omega$, or, geometrically, along any set (in $\Omega$ ) parallel to $\partial \Omega$ (including focal set). In particular, it is true for the distance function (because of (2.9))

$$
\begin{aligned}
& u_{\text {dist }} \in L_{V}^{2}(\Omega), \\
& u_{\text {dist }}(x)=\operatorname{dist}(x, \partial \Omega)=\frac{1}{g}(\arccos \varepsilon-\arccos (V(x))),
\end{aligned}
$$

and (2.22) is equivalent to

$$
\begin{aligned}
L_{V}^{2}(\Omega)=\left\{u \in L^{2}(\Omega):\right. & u=\psi \circ u_{\text {dist }} \text { in } L^{2}(\Omega) \\
& \left.\psi \text { is a function on }\left[0, \frac{1}{g} \arccos \varepsilon\right]\right\} .
\end{aligned}
$$

Any arbitrary function $u \in L_{V}^{2}(\Omega)$ can be approximated by continuous functions from the same subspace. Moreover, because of (2.21), the complete orthogonal basis of $h_{i}, i \geq 1$ in $L_{w}^{2}[\varepsilon, 1]$ gives the complete orthogonal basis of $u_{i}=h_{i} \circ V$ in $L_{V}^{2}(\Omega)$ (we have already known that when level surfaces of $V$ are homogeneous - from Corollary 1, and the basic equations for non-homogeneous level surfaces are actually the same). 
Corollary 2. Let $X$ be a (classical) compact irreducible symmetric space of the first rank, i.e. $X=S^{n}, R P^{n}, C P^{n}$ or $H P^{n}$. Any domain $\Omega \subset X$ with the boundary $\partial \Omega$ being isoparametric hypersurface in $X$, admits solutions (overdetermined eigenfunctions) $u_{i}(x)$ to $(\mathrm{N})$ (respectively, to (D)) for infinitely many eigenvalues $\alpha_{i}>0$. These eigenfunctions, $u_{i}(x)$ (taken once for each $\alpha_{i}$ and with $\alpha_{1}=0, u_{1}(x)$ a constant for $(\mathrm{N}))$, form an orthogonal basis for $L_{V}^{2}(\Omega)$ in (2.22). Any eigenfunction for the first classical Dirichlet eigenvalue is a solution for (D). Also, if there is an overdetermined eigenfunction $u(x)$ (for either (N) or (D)) with an eigenvalue $\alpha=g k(g k+m-1)$ for an integer $k \geq 1\left(m=n\right.$ for $\left.X=S^{n}, R P^{n}\right)$, then $u(x)$ can be extended to a global eigenfunction on $X$.

REMARK. Given a (regular at $V=1$ ) solution $h(V)$ of (2.18) for any sufficiently large $\lambda>0$ (or $\alpha>0$ ), there are zeros $\varepsilon \in(-1,1)$ of $h^{\prime}(V)$ (respectively, of $h(V)$ ) so that the domains $\Omega_{\varepsilon}=\{x \in X: V(x)>\varepsilon\}$ admit solutions to $(\mathrm{N})$ (respectively, to (D)) with the given eigenvalue $\alpha>0$. In fact, for any $\alpha=g k(g k+m-1), k \geq 1$, all zeros of the corresponding Jacobi polynomial (as well as of its derivative which is a multiple of another Jacobi polynomial) belong to $(-1,1)$ (see [Ol]). In particular, there are infinitely many domains $\Omega_{\varepsilon}$ with solutions to $(\mathrm{N})$ (respectively, to (D)) which can be extended to global eigenfunctions on $X$.

Corollary 3. There are spaces $X=S^{n}, R P^{n}, C P^{n}, H P^{n}$ which contain domains $\Omega \subset X$ having non-homogeneous in $X$ boundaries $\partial \Omega$ but admitting solutions to both (N) and (D) for infinitely many eigenvalues $\alpha>0$. In particular, Generalized Schiffer Conjecture (GSC) fails in general for compact irreducible symmetric spaces of the first rank.

As mentioned in Section 0, a domain $\Omega$ fails Pompeiu Property $(\mathrm{PP})$ if $(\mathrm{N})$ has at least one global eigenvalue $\alpha>0$, i.e. there is a solution to $(\mathrm{N})$ in a form of a Jacobi polynomial of $V$. Considering the density of zeros of Legendre polynomials, P. Ungar showed in 1954 (see [U]) that the set of concentric spherical caps in $S^{2}$ (if a center on $S^{2}$ is fixed) has a countable dense subset failing (PP). Now, considering that of Jacobi polynomials (see [Sz]), we obtain a generalization of this fact to any collection of domains whose boundaries form a (continuous) isoparametric family: 
Corollary 4. Let $X$ be a (classical) compact irreducible symmetric space of the first rank, $I \subset R$ an interval and $f(x)$ a non-constant isoparametric function on $X$. Suppose that $\left(M_{\beta}\right), \beta \in I$ is a continuous isoparametric family, with all $M_{\beta}=\{x \in X: f(x)=\beta \in I\}$ being regular (level surfaces may be homogeneous as well as non-homogeneous but they all have the same kind for the given $f)$. Then $\left(M_{\beta}\right)$ has a countable dense subfamily $\left(M_{\beta_{i}}\right), i \geq 1$ such that for each $i$ both domains $\left(\Omega_{\beta_{i}}^{ \pm}\right)$with $\partial \Omega_{\beta_{i}}^{ \pm}=M_{\beta_{i}}$ fail Pompeiu Property (PP).

\section{Domains with isoparametric boundaries in non-symmetric spaces.}

In this section we will construct a number of specific domains $\Omega$ admitting solutions to (N) and (D) in non-symmetric spaces. We will see that even in non-compact spaces boundaries of such domains may still be non-homogeneous. However, metric balls in such spaces, generally speaking, admit solutions neither to (N) nor to (D). For the purposes of this section we need the extension of the theory of isoparametric functions and hypersurfaces to that of isoparametric systems and submanifolds (in a very brief account). We will follow A. West (1993) (see [Wes]).

Definition 4. Let $X$ be a Riemannian manifold and let $f_{1}, \ldots, f_{d}$ be a system of smooth real functions on $X$. Put $F=\left(f_{1}, \ldots, f_{d}\right): X \longrightarrow \mathbb{R}^{d}$. We say that $f_{1}, \ldots, f_{d}$ is an isoparametric system of functions if for all $i, j, k \in\{1,2, \ldots, d\}$ there exist smooth functions $A_{i}, B_{i j}, C_{i j}^{k}$ defined on the image $F(X)$, of $X$ under $F$ so that

$$
\begin{gathered}
\Delta f_{i}=A_{i} \circ F, \\
\left\langle\nabla f_{i}, \nabla f_{j}\right\rangle=B_{i j} \circ F, \\
{\left[\nabla f_{i}, \nabla f_{j}\right]=\sum_{k=1}^{d}\left(C_{i j}^{k} \circ F\right) \nabla f_{k} .}
\end{gathered}
$$

Regular level surfaces of $F$ are called isoparametric submanifolds in $X$.

REMARK. If $X$ is a space of constant sectional curvature, the above definition of an isoparametric submanifold is equivalent to some geo- 
metrical statement about its normal bundle and principal curvatures (C.-L. Terng, 1985) (see [T]) but we will not go into these details.

A. West (1993) (see [Wes]) proved the following:

Proposition 6. Let $X$ be a Riemannian manifold and let $\left(f_{1}, \ldots, f_{d}\right)$ be an isoparametric system. Let $L=f_{d}^{-1}(\tau)$ be a regular level surface of $f_{d}$. Then $\left.f_{1}\right|_{L},\left.f_{2}\right|_{L}, \ldots,\left.f_{d-1}\right|_{L}$ is an isoparametric system on $L$.

In fact, the set of all isoparametric hypersurfaces of $S^{n}$ is the "same" as the set of all compact isoparametric submanifolds of $\mathbb{R}^{n+1}$ of codimension 2 (C.-L. Terng, 1985) (see [T]). That is, the system $F=\left(f, r^{2}\right)$ (where $f(x)$ is a Cartan polynomial and $\left.r=|x|\right)$ is isoparametric on $\mathbb{R}^{n+1}$. Then we can apply Proposition 6 in two possible directions.

In the previous section we have exploited the isoparametricity of $f$ on $S^{n}$ - level surface of $r^{2}$. The other approach is that, according to Proposition $6, r^{2}$ (as well as $r$ ) is an isoparametric function on any regular level surface of $f(x)$ in $\mathbb{R}^{n+1}$.

Now, consider what structure $X=f^{-1}(\tau)$ can have (we assume that $g>1$ since otherwise $X$ is isometric to $\mathbb{R}^{n}$ ). If $\tau=0$, then $X$ contains the origin 0 at which $f$ has a singularity $((2.6)$ implies that $\left.\nabla_{R} f(0)=0\right)$. Thus, in order to have a regular level surface, we will assume that $\tau \neq 0$. Observe that replacing $\tau$ by $-\tau$ we actually replace $f^{-1}(\tau)$ by $(-f)^{-1}(\tau)$. However, if $f(x)$ is a Cartan polynomial with the parameters $g, m_{0}, m_{1}$, then $-f(x)$ is another Cartan polynomial with parameters $g, m_{1}, m_{0}$, i.e. with $m_{0}$ and $m_{1}$ switched. (As mentioned above, Cartan polynomial is uniquely defined by $g, m_{0}, m_{1}$ if $m_{1} \neq m_{0}$ and up to \pm if $m_{1}=m_{0}$.) Therefore, without loss of generality, we can assume that $\tau>0$, and, moreover, since $f(x)$ is a homogeneous polynomial, it is enough to take $\tau=1$. Thus, we will consider the Riemannian manifold $X$ of the dimension $n$ being a submanifold of $\mathbb{R}^{n+1}$

$$
X=\left\{x \in \mathbb{R}^{n+1}: f(x)=1\right\}
$$

Any $x \in R^{n+1}$ can be expressed as $x=r \xi, r=|x|, \xi \in S^{n}$ so that the equation

$$
f(x)=1
$$


is equivalent to

$$
f(\xi)=r^{-g}
$$

Since $f\left(S^{n}\right)=[-1,1],(3.6)$ implies that $r \geq 1$ for $x \in X$. Given any $\rho>1$, the level surface

$$
M_{\rho}=\{x \in X: r=\rho\}=\left\{\rho \xi: \xi \in S^{n}, f(\xi)=\rho^{-g}\right\}
$$

is regular, i.e. $M_{\rho}$ is an isoparametric hypersurface in $X$. Of course, $M_{\rho}$ is similar to an isoparametric hypersurface in $S^{n}$. If $\rho=1$, then $M_{\rho}=M_{1}=\left\{\xi \in S^{n}: f(\xi)=1\right\}$ is a focal set. Notice, that although $r$ is constant on any $M_{\rho}, M_{\rho}$ is not a metric sphere in $X$ (one could guarantee that only if $X$ is a linear hyperplane of $\mathbb{R}^{n+1}$, i.e. $g=1$ ). Generally, metric spheres in $X$ are neither homogeneous nor isoparametric. $M_{\rho}$ are homogeneous in $X$ if and only if the corresponding hypersurfaces $\widetilde{M}_{\rho}=\left\{\xi \in S^{n}: f(\xi)=\rho^{-g}\right\}$ are homogeneous in $S^{n}$ (all $M_{\rho}$ are either homogeneous or non-homogeneous in $X$ simultaneously). Since we can change $\rho$ continuously from 1 up to $\infty$, we have

$$
X=\bigcup_{\rho \geq 1} M_{\rho}
$$

It follows that $X$ is non-compact, non-symmetric and connected.

The simplest example of $X$ is the hyperboloid given by

$$
f(x)=x_{1}^{2}+\cdots+x_{k}^{2}-x_{k+1}^{2}-\cdots-x_{k+l}^{2}=1,
$$

with $n=k+l-1, k, l>1$ and

$$
M_{\rho}=S^{k-1}\left(\sqrt{\frac{\rho+1}{2}}\right) \times S^{l-1}\left(\sqrt{\frac{\rho-1}{2}}\right) .
$$

Now, for an arbitrary $X$ defined by (3.4) and given any real $a \neq 0$ (not necessarily integer), we can compute (omit the calculations here) that

$$
\left\{\begin{array}{c}
\Delta\left(r^{a}\right)=a r^{a-2}\left(n+a-2+(g-a+1) r^{-2 g}\right. \\
\left.-\frac{m_{1}-m_{0}}{2} g r^{-g}\right) \\
\left\|\nabla\left(r^{a}\right)\right\|^{2}=a^{2} r^{2 a-2}\left(1-r^{-2 g}\right)
\end{array}\right.
$$


where $\Delta=\Delta_{X}, \nabla=\nabla_{X}$.

Now we are ready to solve (N) and (D) for domains $\Omega \subset X$ with the boundaries $\partial \Omega$ which are isoparametric in $X$. Indeed, $n-1=$ $g\left(m_{1}+m_{0}\right) / 2$ and $(2.16),(2.17),(3.9)$ yield

Theorem 4. Let $X$ be a Riemannian manifold as in (3.4), with a Cartan polynomial $f(x)$ of degree $g>1$. Then, given $\rho>1, \Omega=\{x \in$ $X: \quad r=|x|<\rho\}$ admits solutions to both (N) and (D) for infinitely many eigenvalues $\alpha>0$. These solutions have the form $u(x)=h(r)$ where $h(r)$ is a solution of the equation

$$
\begin{aligned}
\left(1-r^{-2 g}\right) h^{\prime \prime}(r)+g & \left(\frac{m_{1}+m_{0}}{2} r^{-1}+r^{-2 g-1}\right. \\
& \left.\quad-\frac{m_{1}-m_{0}}{2} r^{-g-1}\right) h^{\prime}(r)+\alpha h(r)=0,
\end{aligned}
$$

with $h(r)$ regular at $r=1$ and $h^{\prime}(\rho)=0$ for $(\mathrm{N})$ or $h(\rho)=0$ for $(\mathrm{D})$. In particular, any eigenfunction for the first classical Dirichlet eigenvalue is a solution for $(\mathrm{D})$. Also, the above solutions can be extended to global eigenfunctions on $X$.

Remark. Defining

$$
X_{\varepsilon}=\left\{x \in \mathbb{R}^{n+1}: \tilde{f}(x)=1\right\}, \quad \tilde{f}(x)=|x|^{g}+\varepsilon f(x),
$$

where $\varepsilon \in(-1,1)$ is a constant, we will get the compact non-symmetric space $X_{\varepsilon}$ with $r$ still isoparametric on it. The domains $\Omega$ constructed in a way similar to that in Theorem 4 will admit solutions to (N) and (D) for infinitely many eigenvalues (but not necessarily extendible to the whole $X_{\varepsilon}$ ). In particular, if $g=2$ and $f(x)$ is a quadratic polynomial in (3.8) and $X_{\varepsilon}$ is just the surface of an ellipsoid with exactly two different main axes - in this case domains $\Omega$, admitting solutions to (N) and (D), have the boundaries of a kind $S^{k-1}\left(r_{1}\right) \times S^{l-1}\left(r_{2}\right)$, i.e. well familiar Generalized Clifford Torus.

In fact, we can consider $X_{\varepsilon}$ as a continuous deformation of $X_{0}=S^{n}$ as $\varepsilon$ is changing. It is interesting that such a deformation preserves solvability of (N) and (D) for a certain class of domains in $S^{n}$, namely, for the domains whose boundaries are level surfaces of $f$ in $S^{n}$. A related result when $X$ is $\mathbb{R}^{n}$ or non-compact irreducible symmetric spaces of the first rank was obtained by M. Agranovsky and A. Semenov in 1996 
(see $[\mathrm{AS}]$ ). They proved that if a continuous deformation of a metric ball as a domain $\Omega$ preserves the solvability of $(\mathrm{N})$ with a bounded eigenvalue, then $\Omega$ must remain a ball during the deformation (with center and radius possibly changing). The key difference with our situation is that we consider the deformation of a space $X_{0}=S^{n}$ (embedded into $\mathbb{R}^{n+1}$ ), namely, $X_{\varepsilon}$ (not remaining a sphere!) defined by (3.11), which, as we said, preserves solvability of (N) and (D) for some domains $\Omega \subset S^{n}=X_{0}$ (but not for all of them). In particular, one can arrange a deformation in such a way that the boundary $\partial \Omega$ remains unchanged (in $\mathbb{R}^{n+1}$ ) while $\Omega$ itself is changing along with $X$ - and the solvability is still preserved.

Corollary 5. There are non-compact irreducible non-symmetric spaces $X$ which contain domains $\Omega \subset X$ having non-homogeneous in $X$ boundaries $\partial \Omega$ but admitting solutions to both (N) and (D) for infinitely many eigenvalues $\alpha>0$. In particular, Generalized Schiffer Conjecture (GSC) fails in general for non-compact irreducible non-symmetric spaces.

REMARK. As follows from the remark after Theorem 4, a number of compact irreducible non-symmetric spaces containing domains with the same properties as in Corollary 5, can be constructed as well.

\section{Domains with isoparametric boundaries in arbitrary Rie- mannian manifolds.}

\subsection{Geometrical realization of a singular ordinary differential equation.}

Suppose we are given smooth functions $p(t)$ and $q(t)$ with $p(t)>0$ on $[a, b)$ and $p(b)=0$. One can ask, does there exist a Riemannian manifold $X$ and an isoparametric function $V$ on $X$ with the maximum value $b$, such that

$$
\|\nabla V(x)\|^{2}=p(V(x)), \quad \Delta V(x)=q(V(x)),
$$

and $\Omega=\{x \in X: V(x)>a\}$ is relatively compact and connected as well as its boundary $\partial \Omega=\{x \in X: V(x)=a\}$ ?

This question is interesting because when the answer is positive, the eigenvalue problem for ordinary differentail equations with singularity 
at $t=b$

$$
\begin{aligned}
& p(t) h^{\prime \prime}(t)+q(t) h^{\prime}(t)+\alpha h(t)=0, \\
& t \in(a, b), h^{\prime}(a)=0, \text { or } h(a)=0,
\end{aligned}
$$

is equivalent to an overdetermined boundary value problem $(\mathrm{N})($ or $(\mathrm{D}))$ with $u(x)=h(V(x))$ and

$$
\begin{aligned}
& \Delta h(V(x))+\alpha h(V(x)) \\
& =\|\nabla V(x)\|^{2} h^{\prime \prime}(V(x))+\Delta V(x) \cdot h^{\prime}(V(x))+\alpha h(V(x)) \\
& =p(V(x)) h^{\prime \prime}(V(x))+q(V(x)) h^{\prime}(V(x))+\alpha h(V(x)) .
\end{aligned}
$$

Definition 5. We call a triple of $X, \Omega \subset X$ and $V(x)$ a geometrical realization of a singular ordinary differential equation (4.2) if the above conditions are satisfied.

Geometrical realization conjecture. A geometrical realization for (4.2) exists at least if both $p(t)$ and $q(t)$ are real analytic, $p(t)>0$ on $[a, b)$, singularity of this ordinary differential equation at $t=b$ is simple $\left(p^{\prime}(b) \neq 0\right)$, and there exists a complete system of regular eigenfunctions for (4.2).

REMARK. In fact, given a continuous isoparametric family in a Riemannian manifold $X, q(b) / p^{\prime}(b)$ is an invariant of the family, not depending on the choice of $a, b$ and the function $V(x)$ in (4.1) $(p(t)$ and $q(t)$ are uniquely defined by $V$ ). In all examples considered in this paper, $2 q(b) / p^{\prime}(b)$ is an integer (actually, $m_{0}+1$ ) but the author believes this is not necessary.

\subsection{Solvability of $(\mathrm{N})$ and (D).}

Conversely, suppose $X$ is an arbitrary Riemannian manifold, $\Omega \subset$ $X$ is a bounded domain with connected boundary $\partial \Omega$ and $V(x)$ is an isoparametric function on $X$ (or at least in a neighborhood of $\bar{\Omega}$ ), satisfying (4.1) with $p(t) \geq 0$ and $\partial \Omega$ being a regular connected component of a level set of $V$. We assume that $V(x)$ is real analytic on $X$, which implies that $p(t)$ and $q(t)$ are both continuous on $V(X) \subset R$ (or $V(\bar{\Omega}) \subset R$ ) and analytic on $V(X) \backslash E($ or $V(\bar{\Omega}) \backslash E$ ) where $E$ is the 
set of zeros of $p(t)$. We don't know for sure what kind of singularities $p(t)$ and $q(t)$ may have on $E$. In general, for non-homogeneous isoparametric boundaries, it is not easy to say whether (N) or (D) (with, of course, $u(x)=h(V(x)))$, or, which is the same, singular ordinary differential equation (4.2) has a solution (eigenvalue). The key point of analysis in each particular case is that for Sobolev spaces (see $[R]$ ) of functions $u(x)$ on $\Omega$, the property of being constant along level sets of $V$ is preserved under the operations $u \longrightarrow \Delta u$ and $u, v \longrightarrow\langle\nabla u, \nabla v\rangle$ in view of (4.1). In the subspaces of functions with that property, the Fredholm alternative (see $[R]$ ) implies the existence of countably many eigenvalues $\alpha$ and corresponding eigenfunctions $u(x)$ but the main difficulty is the behavior of $u(x)$ on the focal sets of $V(x)$ corresponding to the zeros of $p(t)(V(x) \in E$ if and only if $\nabla V(x)=0)$.

In many important cases (like in those considered in this paper), if $p(t)>0$ on $[a, b)$ and $b$ is a regular zero of $p(t)$, then, under certain conditions, (4.2) has a complete system of solutions and so does (N) $($ or $(\mathrm{D}))$.

\section{Other overdetermined boundary value problems and appli- cations.}

The approach described above can be extended to a number of other (essentially more complicated) overdetermined boundary value problems. Below $V(x)$ will denote an isoparametric function on a Riemannian manifold $X$ so that when we choose a domain $\Omega \subset X$ with an isoparametric boundary, we mean that such a boundary is a level surface of $V$, i.e.

$$
\Omega=\{x \in X: V(x)>s\}, \quad \partial \Omega=\{x \in X: V(x)=s\},
$$

or

$$
\Omega=\{x \in X: V(x)<s\}, \quad \partial \Omega=\{x \in X: V(x)=s\},
$$

where $s$ is some constant. 


\subsection{Higher order equations.}

Typically, a linear higher order overdetermined boundary value problem has a form

$$
\begin{cases}\Delta^{m} u+a_{1} \Delta^{m-1} u+\cdots+a_{m-1} \Delta u+\alpha u=0, & \text { in } \Omega, \\ u=c_{0}, \frac{\partial u}{\partial n}=c_{1}, \frac{\partial^{2} u}{\partial n^{2}}=c_{2}, \ldots & \text { on } \partial \Omega,\end{cases}
$$

where $a_{1}, a_{2}, \ldots, a_{m-1}$ are coefficients (constants), $\alpha$ an eigenvalue, $c_{0}, c_{1}, \ldots$ are constants whose number i.e., the number of conditions of the kind $\partial^{k} u / \partial n^{k}=c_{k}$, is bigger than $m$ while $0 \leq k \leq 2 m-1$ for all such $k$ and some of $c_{k}$ (but not all) may equal zero or be unknown. The simplest example is $m=2$, i.e. biharmonic equation (when $a_{1}=0$ ), which is well known in the theory of thin membrane oscillations.

The question, as before, is whether the existence of a solution to (5.1) implies that $\Omega$ is a ball (or $\partial \Omega$ is homogeneous in $X$ ). Choosing $\Omega$ with $\partial \Omega$ isoparametric in $X$ we will get a number of domains admitting solutions to (5.1) in form $u=h(V)$ and therefore giving the negative answer to that question in general (unless $X$ is $\mathbb{R}^{n}$ or non-compact irreducible symmetric spaces of the first rank).

REMARK. Of course, overdetermined boundary value problems can be considered for the system of differential equations (of the second or higher order) as well.

\subsection{Nonlinear equations.}

Many applied problems lead to nonlinear equations, in particular, a number of overdetermined boundary value problems for such equations have a form

$$
\begin{cases}F\left(u,|\nabla u|^{2}, \Delta u, \nabla u \cdot \operatorname{Hess}(u) \cdot \nabla u\right)=0, & \text { in } \Omega, \\ u=c_{0}, \frac{\partial u}{\partial n}=c_{1}, & \text { on } \partial \Omega .\end{cases}
$$

Here $\nabla u \cdot \operatorname{Hess}(u) \cdot \nabla u=\nabla u \cdot \nabla\left(|\nabla u|^{2}\right) / 2$ and in the case of $X=\mathbb{R}^{n}$

$$
\nabla u \cdot \operatorname{Hess}(u) \cdot \nabla u=\sum_{i, j=1}^{n} \frac{\partial u}{\partial x_{i}} \frac{\partial^{2} u}{\partial x_{i} \partial x_{j}} \frac{\partial u}{\partial x_{j}}, \quad x \in \mathbb{R}^{n} .
$$


An example of nonlinear equation in (5.2) is the equation of gas dynamics, such as the stationary irrotational flow of an ideal compressible fluid described by the equation of continuity which can be written in the divergence form (see $[\mathrm{GiT}]$ )

$$
\operatorname{div}(\rho(|\nabla u|) \nabla u)=0
$$

where $u$ is the velocity potential of the flow and the fluid density $\rho$ satisfies a density-speed relation $\rho=\rho(|\nabla u|)$. At the present time, the only known domains admitting solutions to (5.2) in $\mathbb{R}^{n}$ are metric balls (but, of course, not for any nonlinear problem does such a solution exist). For some elliptic problems of a form (5.2) in $\mathbb{R}^{n}$ it is proven that if $\Omega$ admits a solution (with some conditions, for instance, $u \geq 0$ ), then it must be a ball, e.g. (see [S]) and further developments in (see $[\mathrm{GaL}])$. It is important to note that in the case of nonlinear problems, homogeneity of $\partial \Omega$ (in any space) doesn't automatically imply the existence of such solutions. In the spaces considered above (for Laplace-Beltrami operator) we can choose the domains $\Omega$ with isoparametric boundaries and try to find the solutions in form $u=h(V)$. Of course, the simplest isoparametric hypersurfaces are metric spheres and Generalized Clifford Tori (which, in some sense, can be considered as surfaces of revolution). As we know, given a space $X$, for different kinds of isoparametric boundaries $\partial \Omega$ ordinary differential equation on $h$ are very similar. Thus, existence of a radially symmetric solution to (5.2) (for $\Omega$ balls or $\partial \Omega$ Generalized Clifford Tori) often implies existence of solutions to (5.2) for $\Omega$ with other kinds of isoparametric boundaries $\partial \Omega$ (maybe, with $F$ in (5.2) replaced by a slightly different $\widetilde{F})$. Moreover, if $\partial \Omega$ is isoparametric, then so is the distance function $u_{\text {dist }}(x)=\operatorname{dist}(x, \partial \Omega)$ (except, maybe, focal points) and the above approach can allow us to find solutions to a more general overdetermined boundary value problem, namely

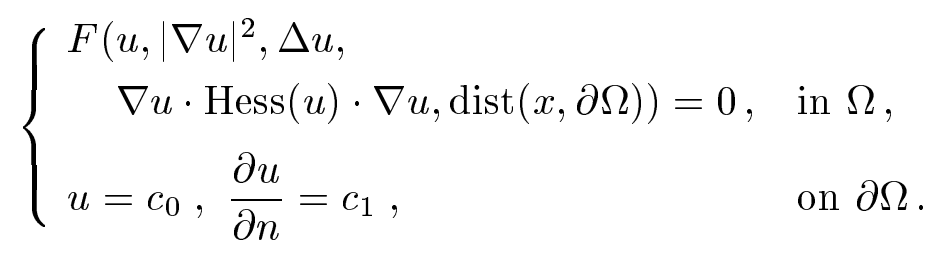

A simpler case is an equation of the form

$$
\Delta u+F\left(u,|\nabla u|^{2}\right)=0
$$


with $F(0,0)=0$. Let

$$
\alpha=\left.\frac{\partial F}{\partial u}\right|_{u=0, \nabla u=0}, \quad \varphi\left(u,|\nabla u|^{2}\right)=F\left(u,|\nabla u|^{2}\right)-\alpha u,
$$

so that

$$
\varphi(0,0)=0,\left.\quad \frac{\partial \varphi}{\partial u}\right|_{u=0, \nabla u=0}=0
$$

and Taylor expansion at $(0,0)$ has a form

$$
\varphi\left(u,|\nabla u|^{2}\right)=a_{0,1}|\nabla u|^{2}+a_{2,0} u^{2}+\cdots
$$

Then we can consider an overdetermined boundary value problem

$$
\begin{cases}\Delta u+\varphi\left(u,|\nabla u|^{2}\right)+\alpha u=0, & \text { in } \Omega, \\ u=c_{0}, \frac{\partial u}{\partial n}=c_{1}, & \text { on } \partial \Omega .\end{cases}
$$

When $\max |u(x)|, \max |\nabla u(x)|$ on $\bar{\Omega}$ are sufficiently small, we can consider (5.4) as a perturbation of a linear problem (see [R]) (obtained by omitting $\varphi\left(u,|\nabla u|^{2}\right)$ ) and this guarantees the existence of solutions (in form $u=h(V)$ with $\partial \Omega$ isoparametric) for a number of important cases.

Of course, the above consideration can be extended to overdetermined boundary value problems for systems of differential equations and for higher order nonlinear equations involving invariant differential operators, such as $|\nabla u|^{2}, \Delta u, \nabla u \cdot \operatorname{Hess}(u) \cdot \nabla u, \ldots$ and their various combinations and compositions.

\section{Linear time-dependent equations.}

It is interesting to consider initial value problem for linear heat equation with the classical Dirichlet condition on the boundary.

$$
\begin{cases}\frac{\partial u}{\partial t}=a^{2} \Delta u, & t \geq 0, x \in \Omega, \\ u=c_{0}, & t=0, x \in \Omega, \\ u=0, & t>0, x \in \partial \Omega,\end{cases}
$$

where $a>0, c_{0} \neq 0$ are constants. A solution $u(x, t)$ to $(5.5)$ exists and it is unique. It can be found, say, by separation of variable as

$$
u(x, t)=\sum_{k=1}^{\infty} u_{k}(x) e^{-a^{2} \alpha_{k} t},
$$


where $u_{k}(x)$ are Dirichlet eigenfunctions (some $u_{k}(x)$ can be zero, but there must be infinitely many non-zero terms). The question is the following: given a solution, suppose $\partial u / \partial n$ at $\partial \Omega$, i.e. boundary heat flow (up to a constant coefficient) is constant at any fixed moment $t$ (i.e., depends only on $t>0$ and doesn't depend on $x \in \partial \Omega$ ). Does it imply that $\Omega$ is a ball (or, generally, that $\partial \Omega$ is homogeneous in $X$ )? In $\mathbb{R}^{n}$, it does. Indeed, in view of $(5.6)$ it would follow that all $u_{k}(x)$ are constant on $\partial \Omega$, i.e. are solutions to overdetermined Dirichlet Problem (D). Now, without loss of generality assume that $c_{0}=1$ in (5.5). Then (5.6) implies at $t=0$

$$
1=\sum_{k=1}^{\infty} u_{k}(x)
$$

where eigenfunctions are $u_{k}(x)$ orthogonal to one another in $L^{2}(\Omega)$. Similarly to the proof of Lemma 2, one can easily see that overdetermined Dirichlet problem (D) has a solution for the first eigenvalue (any first classical Dirichlet eigenfunction has a constant sign in $\Omega$ ). This forces $\Omega$ to be a ball and solutions to be radially symmetric. But, for the spaces considered above for Laplace-Beltrami operator, we can choose $\Omega$ to have isoparametric boundary and find the solution of (5.5) in form

$$
u(x, t)=h(V(x), t)=\sum_{k=1}^{\infty} h_{k}(V(x)) e^{-a^{2} \alpha_{k} t}
$$

and $h_{k}(V)$ are regular solutions of the corresponding ordinary differential equation. Then $\partial u /\left.\partial n\right|_{\partial \Omega}$ will not depend on $x \in \partial \Omega$. (This is true for restriction of $u(x, t)$ on any isoparametric level surface of $V(x)$ or focal set.)

Of course, the same consideration applies to linear wave equation

$$
\begin{cases}\frac{\partial^{2} u}{\partial t^{2}}=a^{2} \Delta u, & t \geq 0, x \in \Omega, \\ u=c_{0}, \frac{\partial u}{\partial t}=c_{1}, & t=0, x \in \Omega, \\ u=0, & t>0, x \in \partial \Omega,\end{cases}
$$

where $a>0, c_{0}, c_{1}$ are constant with

$$
c_{0}^{2}+c_{1}^{2}>0
$$


A special kind of linear wave equation on $S^{n}$ (not initial/boundary value problem), namely

$$
\frac{\partial^{2} u}{\partial t^{2}}-\Delta_{S} u+\left(\frac{n-1}{2}\right)^{2} u=0, \quad t \in \mathbb{R}, x \in S^{n}
$$

was considered by J. Kaneko in 1988 (see [Kan]). He studied the existence of a simple progressive wave solution (SPW) for (5.10) such that SPW's fronts are isoparametric hypersurfaces having three or more distinct principal curvatures with the same multiplicity $m_{1}=m_{0}=m=1$ or 2. That is, he considered the existence of a solution in the form

$$
u(x, t)=U(x) f\left(t-\frac{1}{g} \arccos (V(x))\right),
$$

where $U(x)$ is a specific function on $S^{n}, f$ is an arbitrary function on $R$ and $V(x)$ is the resriction of a Cartan polynomial on $S^{n} \subset \mathbb{R}^{n+1}$ (with the degree $g \geq 3$ and $m=1$ or 2 ). Kaneko proved that SPW doesn't exist if $m=1$ (when isoparametric hypersurfaces are necessarily homogeneous) but exists if $m=2$, in which case $U(x)$ in (5.11) must be constant. Unlike Kaneko's conclusions on SPW, the results on initial/boundary value problems considered in this paper (about the existence) don't depend on whether multiplicities are equal, how big they are and whether isoparametric hypersurfaces are homogeneous or not. Moreover, the principal curvatures of isoparametric hypersurfaces can be non-constant (in spaces different from $\mathbb{R}^{n}, S^{n}, R P^{n}$ or $H^{n}$ ) and the existence still holds.

A number of linear higher order time-dependent problems or problems involving systems of equations can also be considered in the same way as for (5.5) or (5.9).

\subsection{Nonlinear time-dependent equations.}

Consider nonlinear initial/boundary value problem for heat type equation

$$
\begin{cases}\frac{\partial u}{\partial t}=F\left(u,|\nabla u|^{2}, \Delta u, \nabla u \cdot \operatorname{Hess}(u) \cdot \nabla u\right), & t \geq 0, x \in \Omega \\ u=c_{0}, & t=0, x \in \Omega \\ \frac{\partial u}{\partial n}=0(\text { or } u=0), & t>0, x \in \partial \Omega\end{cases}
$$


or for wave type equation

$$
\left\{\begin{aligned}
\frac{\partial^{2} u}{\partial t^{2}}=F\left(u,|\nabla u|^{2}, \Delta u,\right. & \\
\nabla u \cdot \operatorname{Hess}(u) \cdot \nabla u), & t \geq 0, x \in \Omega, \\
u=c_{0}, \frac{\partial u}{\partial t}=c_{1}, & t=0, x \in \Omega, \\
\left.\frac{\partial u}{\partial n}=0 \text { (or } u=0\right), & t>0, x \in \partial \Omega .
\end{aligned}\right.
$$

(Initial/boundary value problems for systems of nonlinear time-dependent equations or for higher order equations can also be considered.) In a number of important cases, it is possible to prove the existence of a solution to (5.12) or (5.13) for sufficiently small $t \geq 0$ (at least). However, it often happens that the solution can not exist for indefinite period of time. That is, after some time blow-up (or, maybe, other kind of singularity) can occur. In general, this time (call it $t_{b u}$ ) is different for different $x \in \bar{\Omega}$, i.e.

$$
t_{b u}=t_{b u}(x), \quad x \in \bar{\Omega}
$$

and the distribution of the blow-up time on $\bar{\Omega}$ is often important. One can say that the domain of $u(x, t)$ is a subdomain of $(x \in \bar{\Omega}) \times(t \geq 0)$ defined by

$$
0 \leq t<t_{b u}(x)
$$

(for some points $x \in \bar{\Omega}, t_{b u}(x)$ may be infinite). In particular, it is interesting to know how $t_{b u}$ is distributed on the boundary $\partial \Omega$. In the case of radial symmetry, when $\Omega$ is a ball in $\mathbb{R}^{n}$, the solution is radially symmetric and $t_{b u}$ is constant on $\partial \Omega$ (as well as on any sphere concentric with $\partial \Omega$ ). It would be hard (if not impossible) to find domains $\Omega \subset \mathbb{R}^{n}$ (non-balls) with $t_{b u}(x)$ constant on $\partial \Omega$.

In the spaces $X$ considered in this work, the theory developed for the domains $\Omega$ with isoparametric boundaries $\partial \Omega$ (being level surfaces of $V(x))$ allows us to find a solution $u(x, t)$ for $(5.12)$ or $(5.13)$ in a form

$$
u(x, t)=h(V(x), t) .
$$

Like that in the above problems, $\partial \Omega$ can be non-homogeneous in $X$ : if such a solution exists for a similar problem with $\partial \Omega$ radially symmetric (say, being metric sphere or Generalized Clifford Torus), then it 
exists for domains $\Omega \subset X$ with $\partial \Omega$ being other kinds of isoparametric hypersurfaces as well. The blow-up for $u(x, t)$, if occurs, comes at the same time to any level surface of $V(x)$. This time is uniquely defined by the level surface, i.e., by the value of $V$ (i.e., $\left.t_{b u}=t_{b u}(V(x))\right)$. In particular, the blow-up comes at the same time to the boundary $\partial \Omega$. Also, the time of blow-up is the same for all points of the focal set.

\section{Conclusion.}

We have considered a number of compact irreducible symmetric spaces and non-compact irreducible non-symmetric spaces (similarly to the latter, a number of compact irreducible non-symmetric spaces can be considered). These spaces contain domains $\Omega$ admitting solutions to $(\mathrm{N})$ and (D), even for infinitely many eigenvalues $\alpha>0$. The boundaries $\partial \Omega$ of such domains (constructed in this work) are isoparametric hypersurfaces which may be homogeneous as well as non-homogeneous (metric balls not always necessarily admit solutions to (N) and (D)). In particular, the Generalized Schiffer Conjecture and even its weaker version fail in the above spaces.

It follows that in compact irreducible symmetric spaces of the first rank, any collection of domains whose boundaries form an isoparametric family, has a countable dense subcollection of domains failing the Pompeiu Property (which extends the result of P. Ungar for concentric spherical caps in $S^{2}$ ).

We also showed that there are deformations of $S^{n}$ in $\mathbb{R}^{n+1}$ preserving the solvability of (N) and (D) for certain classes of domains $\Omega \subset S^{n}$.

We have discussed the solvability of (N) and (D) for domains with isoparametric boundaries in arbitrary Riemannian Manifolds. While the domains with homogeneous boundaries always admit solutions to $(\mathrm{N})$ and (D), in general, for non-homogeneous isoparametric boundaries, it is not easy to say whether $(\mathrm{N})$ or $(\mathrm{D})$, or, which is the same, the corresponding singular ordinary differential equation (4.2) has a solution (eigenvalue). Nevertheless, in many important cases, if the singularity of (4.3) is simple, then, under certain conditions, (4.2) has a complete system of solutions and so does (N) (or (D)). Our Geometrical Realization Conjecture suggests that, if an arbitrary ordinary differential equation (4.2) has a simple singularity and a complete system of regular eigenfunctions, then, under certain conditions, there is a domain 
$\Omega$ in a Riemannian manifold $X$ with the boundary $\partial \Omega$ isoparametric in $X$ and such that $(\mathrm{N})$ and (D) can be reduced to (4.2).

Finally, the developed approach can be extended to other (essentially more complicated) overdetermined boundary value problems, including higher order equations and non-linear equations, which, in a number of important cases, may also have solutions in domains with isoparametric (and not necessarily homogeneous) boundaries. Also, a number of initial/boundary value problems for time-dependent equations with some extra boundary conditions (say, for the solution $u(x, t)$ on $\partial \Omega$, to depend only on $t$ but not on $x \in \partial \Omega$ ), have solutions for domains with the above boundaries. If a time-dependent equation is non-linear and has blow-up, this blow-up occurs at the same time at all the points on the boundary.

\section{References.}

[AS] Agranovsky, M., Semenov, A., Deformations of balls in Schiffer's Conjecture for Riemannian symmetric spaces. Israel J. Math. 95 (1996), 43-59.

[Be] Berenstein, C. A., An inverse spectral theorem and its applications to the Pompeiu Problem. J. Anal. Math. 37 (1980), 128-144.

[BeS] Berenstein, C. A., Shahshahani, M., Harmonic analysis and the Pompeiu problem. Amer. J. Math. 105 (1983), 1217-1229.

[BeY] Berenstein, C. A., Yang, P., An inverse Neumann problem. J. reine angew. Math. 382 (1987), 1-21.

[Carm] do Carmo, M. P., Riemannian Geometry. Birkhauser, 1992.

[Cart] Cartan, E., Families de surfaces isoparametrique dans les space a courbure constante. Annali di Mat. 17 (1938), 177-191.

[Ch] Chavel, I., Eigenvalues in Riemannian Geometry. Academic Press, 1984.

[GaL] Garofalo, N., Lewis, J., A symmetry result related to some overdetermined boundary value problems. Amer. J. Math. 111 (1989), 9-33.

[GiT] Gilbarg, D., Trudinger, N. S., Elliptic Partial Differential Equations of Second Order. Springer-Verlag, 1983.

[H1] Helgason, S., Differential Geometry and Symmetric Spaces. Academic Press, 1962.

[H2] Helgason, S., Groups and Geometric Analysis. Academic Press, 1984.

[Kan] Kaneko, J., Wave equation and isoparametric hypersurfaces. Geometry of manifolds (Matsumoto, 1988), 165-180. Perspect. Math. 8 Academic Press, 1989. 
[Kar] Karlovitz, M. A., Some solutions to overdetermined boundary value problems on subdomains of spheres. Ph. D. Thesis. Univ. of Maryland, 1990.

[KiN] Kinderlehrer, D., Nirenberg, L., Regularity in free boundary problems. Ann. Scuola Norm. Sup. Pisa Cl. Sci. 4 (1977), 373-391.

[Mul] Mullen, S., Isoparametric systems on symmetric spaces. Geometry and topology of submanifolds. VI (Leuven, 1993/Brussels, 1993), 152-154. World Sci. Publishing, 1994.

[Mun] Munzer, H. F., Isoparametrische hyperflachen in spharen. I; II. Math. Ann. 251 (1980), 57-71. 256 (1981), 215-232.

[Mo] Molzon, R., Symmetry and overdetermined boundary value problems. Forum Math. 3 (1991), 143-156.

[Ol] Olver, F. W. J., Asymptotics and Special Functions. A K Peters, Ltd., 1997.

[OzT] Ozeki, H., Takeuchi, M., On some types of isoparametric hypersurfaces in spheres. I; II. Tohoku Math. J. 27 (1975), 515-559. 28 (1976), 7-55.

[Q1] Qiming, W., Isoparametric maps of Riemannian manifolds and their applications. Advances in Science of China Mathematics. 2 (1986), 79-103.

[Q2] Qiming, W., Isoparametric hypersurfaces in complex projective spaces. Proceedings of the 1980 Beijing Symposium on Differential Geometry and Differential equations. 1, 2,3 (1980), 1509-1523. Science Press, 1982.

[R] Renardy, M., Rogers, R., An Introduction to Partial Differential Equations. Springer-Verlag, 1993.

[Se] Serrin, J., A symmetry problem in potential theory. Arch. Rational Mech. Anal. 43 (1971), 304-318.

[Sh] Shklover, V., Areas of isoparametric hypersurfaces and volumes bounded by them. Preprint, 1999.

[Sz] Szego, G., Orthogonal polynomials. Fourth edition. American Mathematical Society, Colloquium Publications XXIII. American Mathematical Society, 1975.

[T] Terng, C.-L., Isoparametric submanifolds and their Coxeter groups. J. Differential Geom. 21 (1985), 79-107.

[U] Ungar, P., Freak theorem about functions on a sphere. J. London Math. Soc. 29 (1954), 100-103.

[Wei] Weinberger, H. F., Remark on the preceding paper of Serrin. Arch. Rational Mech. Anal. 43 (1971), 319-320. 
[Wes] West, A., Isoparametric systems on symmetric spaces. Geometry and topology of submanifolds, V (Leuven/Brussels, 1992), 281-287. World Sci. Publishing, 1993.

[Wi] Williams, S. A., Analyticity of the boundary for Lipschitz domains without the Pompeiu Property. Indiana Univ. Math. J. 30 (1981), 357-369.

Recibido: 24 de febrero de 1.999

Revisado: 1 de septiembre de 1.999

Vladimir E. Shklover Department of Mathematics

University of Maryland

College Park

MD 20742, USA

ves@math . nwu . edu 